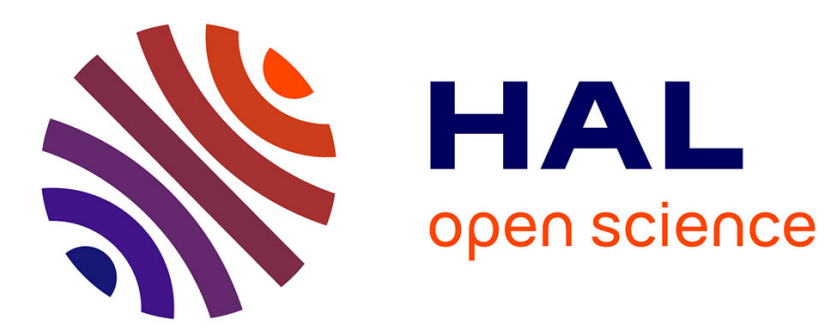

\title{
HOMEOMORPHISM OF HEREDITARILY LOCALLY CONNECTED CONTINUA
}

Aymen Daghar

\section{To cite this version:}

Aymen Daghar. HOMEOMORPHISM OF HEREDITARILY LOCALLY CONNECTED CONTINUA. 2021. hal-03223435v2

\section{HAL Id: hal-03223435 \\ https://hal.science/hal-03223435v2}

Preprint submitted on 24 May 2021

HAL is a multi-disciplinary open access archive for the deposit and dissemination of scientific research documents, whether they are published or not. The documents may come from teaching and research institutions in France or abroad, or from public or private research centers.
L'archive ouverte pluridisciplinaire HAL, est destinée au dépôt et à la diffusion de documents scientifiques de niveau recherche, publiés ou non, émanant des établissements d'enseignement et de recherche français ou étrangers, des laboratoires publics ou privés. 


\title{
HOMEOMORPHISM OF HEREDITARILY LOCALLY CONNECTED CONTINUA
}

\author{
AYMEN DAGHAR
}

\begin{abstract}
Let $f: X \rightarrow X$ be a hereditarily locally connected continuum homeomorphism, we show that any $\omega$-limit set (resp. $\alpha$-limit set) is minimal. Moreover, we show that $\Omega(f)=A P(f)=R(f)$. We also prove that if $P(f)=\emptyset$, then there exists a unique minimal set. On the other hand, if $P(f) \neq \emptyset$ then we prove that any infinite minimal set has the adding machine structure and the absence of Li-Yorke pairs. Consequently, we partially solve the positive entropy conjecture which remains open even in the case of hereditarily locally connected continuum.
\end{abstract}

key word: Minimal set, homeomorphism, hereditarily locally connected continua, $\omega$-limit set, $\alpha$-limit set, nonwandering set, recurrent point.

\section{Introduction}

A continuum is a non-empty compact metric connected set. A continuum is hereditarily locally connected if any subcontinuum of which is locally connected. Hereditarily locally connected are known to be one-dimensional Peano-continua. Note that the class of hereditarily locally connected continua is larger than those of finitely suslinean continua and regular curves (see [8]).

Recently many authors were interested on $\mathbb{Z}$-actions in regular curves. For instance, Seilder [18] proved that those systems have zero topological entropy. Recently Glasner and Megrelishvili [6] proved that they are even null (i.e their sequence topological entropy is zero). In $[14,15]$ Naghmouchi showed that any $\omega$-limit set is minimal and he showed that the absence of periodic point allows the presence for one and only one minimal set. The unique minimal set has been characterized in [4], by showing the existence of an invariant simple closed curve, moreover it was proved that such system does not have Li-Yorke pair. In [3], we proved that infinite $\omega$-limit sets have adding machine structure if we allow periodic points existing and the absence of Li-Yorke pair in this case, thus regular curve homeomorphism does not have Li-Yorke pair. In [11], Mouron showed that a homeomorphism of a suslinean continuum has zero topological entropy. Recently Lipham in [10] highlighted an example of a hereditarily locally connected continuum given by Tymchatyn in [20], which can be approximated by a sequence of mutually disjoint arcs. This re-opens the conjecture of Seidler and Kato

Date: May 24, 2021. 
concerning continua which admit positive entropy homeomorphisms even for the hereditarily locally connected case, which we partially answer in the case of $P(f) \neq \emptyset$.

The class of hereditarily locally connected continua is larger than those of regular curve and finitely suslinean continua. Note that Tymchatyn's example is a hereditarily locally connected continuum, which is neither a regular curve nor a finitely suslinean. This shows that hereditarily locally connected continua could have a more complicated topological structure. We already know that the class of rational curves is larger than the one of hereditarily locally connected continua. Moreover, most of the results proved for regular curves homeomorphisms are false in general for rational curves homeomorphisms. For instance, in [4], it may happen that a rational curve homeomorphism has a positive sequence topological entropy. In [16], the author gave an example of a rational curve homeomorphism which is Li-Yorke chaotic and have an infinite $\omega$-limit set composed exclusively of fixed points.

In the present paper, we deal with hereditarily locally connected continuum homeomorphism and we try to extend the results already proved for regular curves in $([3],[14],[15])$ and to show that hereditarily locally connected continua homeomorphisms share a lot of similarity with the regular curve case.

Plan of the paper: In section 2, we review some known results and give some technical lemmas that will be used later on. In section $3,4,5$ and 6 , we will consider a hereditarily locally connected continuum homeomorphism. In section 3 , we prove that any $\omega$-limit (resp. $\alpha$-limit set) is minimal, we also prove the equality $\Omega(f)=R(f)=A P(f)=\Lambda(f)$. Section 4 will be devoted to the continuity of the maps: $\omega_{f}, \alpha_{f}: X \rightarrow 2^{X}$. In section 5, we consider the case $P(f) \neq \emptyset$ and we prove that $\overline{P(f)}=\Omega(f)$. In section 6 , we prove in the first part that if $P(f)=\emptyset$, then there exists a unique minimal set, which is totally minimal. In the second part, if $P(f) \neq \emptyset$ then we show that any infinite minimal set is an adding machine and the absence of Li-Yorke pair, thus concluding that such homeomorphism has zero topological entropy. At the end of this section, we give a characterization of equicontinoues homeomorphisms of hereditarily locally connected continuum.

\section{Preliminaries}

Let $X$ be a compact metric space. The closure (resp. the boundary) of a subset $A$ of $X$ is denoted by $\bar{A}$ (respectively $\partial(A)$ ), for $C \subset A$, we denote by $B d_{A}(C), C l_{A}(C)$, int $_{A}(C)$ respectively the boundary, the closure and the interior of $C$ in $A$ endowed with the induced topology from the one on $X$. A subset of $X$ is said to be clopen if it is open and closed at the same time. We denote by $2^{X}$ (resp. $\left.C(X)\right)$ the set of all non-empty compact subsets (resp. 
compact connected) subsets of $X$ and we endow $2^{X}$ (resp. $\left.C(X)\right)$ with the Hausdorff metric $d_{H}$ defined as follows:

$$
d_{H}(A, B)=\max \left(\sup _{a \in A} d(a, B), \sup _{b \in B} d(b, A)\right)
$$

where $A, B \in 2^{X}$ and $d(x, M)=\inf _{y \in M} d(x, y)$ for any $x \in X$ and $M \in 2^{X}$. Notice that both $\left(C(X), d_{H}\right)$ and $\left(2^{X}, d_{H}\right)$ are compact metric space (for more details see [13]).

A sequence $\left(A_{n}\right)_{n>0}$ of compact subset of $X$ converges to $A \in 2^{X}$, if $\lim _{n \rightarrow \infty} d_{H}\left(A_{n}, A\right)=0$. Let $A \subset X, \operatorname{diam}(A):=\sup _{x, y \in A} d(x, y)$.

Let $f: X \rightarrow X$ be a continuous map of $X$. We denote by $2^{f}: 2^{X} \rightarrow$ $2^{X}, A \rightarrow f(A)$. Note that $2^{f}$ is a continuous self mapping of $\left(2^{X}, d_{H}\right)$ (see $[13])$.

A dynamical system is a pair $(X, f)$, where $X$ is a compact metric space and $f: X \rightarrow X$ is a continuous map. Let $\mathbb{Z}, \mathbb{Z}_{+}, \mathbb{Z}_{-}$and $\mathbb{N}$ be the sets of integers, non-negative integers, non-positive integers and positive integers, respectively.

Let $(X, f)$ be a dynamical system. The $\omega$-limit set of a given point $x \in X$ is defined as follow:

$$
\begin{gathered}
\omega_{f}(x)=\cap_{n \in \mathbb{N}} \overline{\left\{f^{k}(x): k \geq n\right\}} \\
=\left\{y \in X: \exists n_{1}<n_{2}<\cdots: \lim _{i \rightarrow+\infty} d\left(f^{n_{i}}(x), y\right)=0\right\}
\end{gathered}
$$

If $f$ is a homeomorphism then the full (resp. the backward) orbit under $f$ of a given point $x \in X$ is $O_{f}(x):=\left\{f^{n}(x): n \in \mathbb{Z}\right\}$ (resp. $O_{f}^{-}(x):=$ $\left.\left\{f^{n}(x): n \in \mathbb{Z}_{-}\right\}\right)$. The $\alpha$-limit set of $x$ is $\alpha_{f}(x):=\omega_{f^{-1}}(x)$.

A point $x$ in $X$ is called:

- fixed if $f(x)=x$,

- periodic if $f^{n}(x)=x$ for some $n \in \mathbb{N}$,

- almost periodic if for any neighborhood $U$ of $x$ there exists $N \in \mathbb{N}$ such that $\left\{f^{n+i}(x), i=0,1, \cdots, N\right\} \cap U \neq \emptyset$ for all $n \in \mathbb{Z}_{+}$,

- recurrent if $x \in \omega_{f}(x)$,

- wandering for if there exists neighborhood $U$ of $x$ (called wandering neighborhood ) such that $f^{-n}(U) \cap U=\emptyset$, for every $n \in \mathbb{N}$.

Otherwise, the point $x$ is said to be non-wandering.

We denote respectively by $P(f), A P(f), R(f)$ and $\Omega(f)$, the sets of periodic points, almost periodic points, recurrent points and the non-wandering points of $f$, we also note by $\Lambda(f)=\bigcup_{x \in X} \omega_{f}(x)$. The forward orbit of a given point $x \in X$ is the subset $O_{f^{+}}(x)=\left\{f^{n}(x): n \in \mathbb{Z}_{+}\right\}$. A subset $M$ is called minimal if it is not empty, closed, $f$-invariant (i.e. $f(A) \subset A$ ) and there is no proper subset of $M$ having these properties. A subset $M$ of $X$ is called totally minimal if it is $f^{n}$-minimal for all $n \in \mathbb{N}$. The system $(X, f)$ is said to be transitive if for any two non-empty open sets $U, V$ of $X$ there exists 
$n \in \mathbb{N}$ such that $f^{n}(U) \cap V \neq \emptyset$.

Let $\alpha=\left(j_{1}, j_{2}, \ldots\right)$ be a sequence of integers, where each $j_{i} \geq 2$. Let $\triangle_{\alpha}$ denote the set of all sequences $\left(x_{1}, x_{2}, \ldots\right)$ where $x_{i} \in\left\{0,1, \ldots, j_{i}-1\right\}$ for each $i$. We equip $\triangle_{\alpha}$ by the metric $d_{\alpha}$ given by

$$
d_{\alpha}\left(\left(x_{1}, x_{2}, \ldots\right),\left(y_{1}, y_{2}, \ldots\right)\right)=\sum_{i=1}^{\infty} \frac{\delta\left(x_{i}, y_{i}\right)}{2^{i}}
$$

where $\delta$ is the Kronecker symbol. The addition in $\triangle_{\alpha}$ is defined as follows:

$$
\left(x_{1}, x_{2}, \ldots\right)+\left(y_{1}, y_{2}, \ldots\right):=\left(z_{1}, z_{2}, \ldots\right)
$$

where $z_{1}=\left(x_{1}+y_{1}\right)\left(\bmod j_{1}\right)$ and $z_{2}=\left(x_{2}+y_{2}+t_{1}\right)\left(\bmod j_{2}\right)$, with $t_{1}=0$ if $x_{1}+y_{1}<j_{1}$ and $t_{1}=1$ if $x_{1}+y_{1} \geq j_{1}$. So, we carry a one in the second case. Continue adding and carrying in this way for the whole sequence.

We define $f_{\alpha}: \triangle_{\alpha} \rightarrow \triangle_{\alpha}$ by

$$
f_{\alpha}\left(x_{1}, x_{2}, \ldots\right)=\left(x_{1}, x_{2}, \ldots\right)+(1,0,0, \ldots)
$$

We will refer to the system $\left(\triangle_{\alpha}, f_{\alpha}\right)$ as the adding machine.

Let $A$ be a subset of $X$, we denote by $\Delta_{A}=\{(x, x), x \in A\}$. A pair $(x, y) \in X \times X$ is called proximal if $\liminf _{n \rightarrow \infty} d\left(f^{n}(x), f^{n}(y)\right)=0$ otherwise it is called distal. If $\lim \sup d\left(f^{n}(x), f^{n}(y)\right)=0$, then $(x, y)$ is called asymptotic. A pair $(x, y)$ is called a Li-Yorke pair if it is proximal but not asymptotic. The dynamical system $(X, f)$ is called almost distal if it has no Li-Yorke pair. A pair $(x, y) \in X \times X$ is called regionally proximal if for each $\epsilon>0$ and each pair of open sets $O_{x}, O_{y}$ containing respectively $x$ and $y$, there exist $x^{\prime} \in O_{x}, y^{\prime} \in O_{y}$ and some $n \in \mathbb{N}$ such that $d\left(f^{n}\left(x^{\prime}\right), f^{n}\left(y^{\prime}\right)\right)<\epsilon$.

Denote by $P(X, f)$ the set of proximal pairs, $A(X, f)$ the set of asymptotic pairs and by $R P(X, f)$ the set of regionally proximal pairs. When $f$ is an homeomorphism we denote by $R P(f)=R P(X, f) \cup R P\left(X, f^{-1}\right)$. Clearly $A(X, f), P(X, f)$ and $R P(X, f)$ are $f \times f$ invariant.

Given two dynamical systems $(X, f)$ and $(Y, g)$, by factor map we mean a continuous surjective $\pi: X \rightarrow Y$ satisfying $\pi \circ f=g \circ \pi$, in this case $(X, f)$ is called an extension of $(Y, g)$ and $(Y, g)$ is called a factor of $(X, f)$. Moreover if $\pi$ is an homeomorphism we say that $(X, f)$ and $(Y, g)$ are topologically conjugated.

Recall that a $\mathbb{Z}$-action on $X$ generated by a self homeomorphism $f$ of $X$ is said to be equicontinoues if the family $\left\{f^{n}, n \in \mathbb{Z}\right\}$ is equicontinoues.

Let $(X, f)$ be a dynamical system, where $f$ is a self homeomorphism of $X$, the maximal equicontinous factor $(Y, g)$ of $(X, f)$ is an equicontinous factor of $(X, f)$ such that any equicontinous factor $(Z, h)$ of $(X, f)$ is a factor of $(Y, g)$. 
Lemma 2.1. ([7], Corollary 2.1) Let $(X, f)$ be a dynamical system, where $f$ is a self homeomorphism of $X$. Then the factor $(Y, g)$ induced by the smallest closed invariant equivalence relation containing $R P(f)$ is the maximal equicontinoues factor.

Let $(X, f)$ be a dynamical system, where $f$ is a self homeomorphism of $X$, as a direct consequence of Lemma 2.1 , if $R P(f)=\Delta_{X}$, then $(X, f)$ is equicontinous. For any $x \in X$, denote by $R P_{x}=\{y \in X ;(x, y) \in R P(f)\}$

Lemma 2.2. Let $(X, f)$ be a dynamical system, where $X$ is locally connected continuum and $f$ is a self homeomorphism of $X$, then for any $x \in$ $X, R P_{x}$ is a subcontinuum of $X$.

Proof. Let $x \in X$, it follows immediately by the definition of regionally proximal pair that $R P_{x}$ is closed. Suppose that $R P_{x}$ is not connected then there exists a point $y \in R P_{x} \backslash\{x\}$ such that its connected components $C_{y}$ in $R P_{x}$ is disjoint from the connected component of $x$ in $R P_{x}$.

Claim: There exists an open set $U$ of $X$ such that $R P_{x} \subset U$, in which the connected components of $U$ containing respectively $x$ and $y$ are disjoint.

Indeed, since $R P_{x}$ is a compact subset we can find a sequence of open neighbourhood $\left(O_{n}\right)_{n \geq 0}$ of $R P_{x}$ such that $R P_{x}=\bigcap_{n \geq 0} \overline{O_{n}}$. If for infinitely many $n \geq 0, x, y$ are in the same connected component of $O_{n}$, namely $C_{n}$, then there is a subcontinuum $I \subset R P_{x}$ containing $x$ and $y$ (it suffices to select a suitable converging subsequence of $\left.\left(\overline{C_{n}}\right)_{n>0}\right)$, a contradiction. Hence for some $N$, the connected components of $O_{n}$ containing respectively $x$ and $y$ are disjoint for any $n \geq N$. Consider then $U=O_{N}$. This ends the proof of the claim.

As $(x, y) \in R P(f)$, let $\left(x_{n}\right)_{n \geq 0}$ (resp. $\left.\left(y_{n}\right)_{n \geq 0}\right)$ a sequence of $X$ converging to $x$ (resp. $y$ ) and $\left(m_{n}\right)_{n>0}$ a sequence of integers such that $\lim _{n \rightarrow+\infty} d\left(f^{m_{n}}\left(x_{n}\right), f^{m_{n}}\left(y_{n}\right)\right)=0$. Denote by $C_{x, U}$ and $C_{y, U}$ the connected component of $U$ containing respectively $x$ and $y$, since $U$ is an open set of $X$ which is locally connected and by the claim above, $C_{x, U}$ (resp. $\left.C_{y, U}\right)$ are disjoints open neighbourhood of $x$ (resp. $y$ ), therefore for $n$ large enough, $x_{n} \in C_{x, U}$ and $y_{n} \in C_{y, U}$. Since $\lim _{n \rightarrow+\infty} d\left(f^{m_{n}}\left(x_{n}\right), f^{m_{n}}\left(y_{n}\right)\right)=0$ and $X$ is a locally connected continuum, by Lemma 2.3 we may find a sequence of $\operatorname{arcs}\left(J_{n}\right)_{n \geq 0}$ joining $f^{m_{n}}\left(x_{n}\right)$ and $f^{m_{n}}\left(y_{n}\right)$ such that $\lim _{n \rightarrow+\infty} \operatorname{diam}\left(J_{n}\right)=0$. Observe that for $n$ large enough, $f^{-m_{n}}\left(J_{n}\right)$ is an arc containing $x_{n}$ and $y_{n}$, thus meeting $C_{x, U}$ and $C_{y, U}$. Therefore $f^{-m_{n}}\left(J_{n}\right) \nsubseteq U$, otherwise $C_{x, U}=C_{y, U}$. Hence for some $N \geq 0$ and for any $n \geq N, f^{-m_{n}}\left(J_{n}\right) \cap$ $\partial(U) \neq \emptyset$. Let then $t_{n} \in f^{-m_{n}}\left(J_{n}\right) \cap \partial(U)$, thus $f^{m_{n}}\left(t_{n}\right) \in J_{n}$. It turns out that $\lim _{n \rightarrow+\infty} d\left(f^{m_{n}}\left(x_{n}\right), f^{m_{n}}\left(t_{n}\right)\right)=0$. Let $t \in X$ be some limit point of the sequence $\left(t_{n}\right)_{n \geq N}$, clearly $t \in \partial(U)$, moreover $t \in R P_{x}$, therefore $t \in R P_{x} \cap \partial(U)$ which is a contradiction, since $U$ is an open neighbourhood of $R P_{x}$. 
2.1. On hereditarily locally connected continua. In this subsection we recall and give some topological property of hereditarily locally connected continua.

A family $\left\{A_{i}, i \in I\right\}$ of subset of $X$ is said to be a null family if for any $\epsilon>0$ there exist a finite subset $J$ of $I$ such that for any $i \in I \backslash J$, we have $\operatorname{diam}\left(A_{i}\right)<\epsilon$. A regular curve (resp. a rational curve) is a continuum for which any point has an $\epsilon$-open neighbourhood with finite (resp. at most countable) boundary, for any $\epsilon>0$. A finitely suslinean continuum is a continuum for which any pairwise disjoint family of subcontinua is null. A hereditarily locally connected continuum is a locally connected continuum such that any sub-continuum of which is locally connected, equivalently any connected subset of which is locally connected (see [8], Theorem 9, (v), page 272).

Recall that each regular curve is finitely suslinean and a finitely suslinean continuum is a hereditarily locally connected continuum. Note that a hereditarily locally connected continuum is a locally arcwise connected continuum (for more details see [13] and [8]). The following lemma summarizes some properties of locally connected continua which will be used thought out the paper.

Lemma 2.3. Let $X$ be a locally connected continuum, the following hold: (i) For any $\epsilon>0$, there exists $\eta>0$, such that any two points $x, y \in X$, with $d(x, y)<\eta$ can be joined by an $\operatorname{arc} I$ of $X$ such that $\operatorname{diam}(I)<\epsilon$.

(ii) Any connected open subset of $X$ is arc-wise connected.

(iii) $X$ is supposed to be only a locally connected metric space, given a subset $A$ of $X$ and $C$ some connected component of $A$, then $\partial(C) \subset \partial(A)$.

Proof. For (i) see [8] Theorem 4, page 257 and for (ii) see Theorem 8.26 in [13], for the proof if (iii) see [8] Theorem 3 page 238.

A map $\phi: X \rightarrow Y$ is said to be pointwise monotone if for any $y \in Y$ the set $\phi^{-1}(y)$ is a connected subset of $X$ and monotone if for any connected subset $C$ of $Y \phi^{-1}(C)$ is a connected subset of $X$. Notice that if $\phi$ is onto, then $\phi$ is monotone if and only if it is pointwise monotone (see ([8] Theorem 9, page 131)). Note that if $X, Y$ are continua where $X$ is hereditarily locally connected and $\phi: X \rightarrow Y$ is a continuous onto monotone mapping then $Y$ is also hereditarily locally connected (see [12]).

Definition 2.4. Let $X$ be a continuum. A non degenerate subcontinuum $C$ of $X$ is said to be a convergence continuum if there exists a sequence $\left(C_{n}\right)_{n \geq 0}$ in $C(X)$ that converges to $C$ such that $C \cap C_{n}=\emptyset$, for any $n \geq 0$.

Lemma 2.5. ([8], Theorem 2 page 269) A continuum $X$ is hereditarily locally connected if and only if it contains no convergence continuum $C$.

Lemma 2.6. ([19], Theorem 5) Let $X$ be a hereditarily locally connected continuum and $U$ be an open connected subset of $X$. Then every point $p \in \partial(U)$, is arcwise accessible from $U$ (i.e $U \cup\{p\}$ is arc-wise connected). 
Lemma 2.7. Let $X$ be a hereditarily locally connected continuum. A family $\mathcal{C}$ of subsets of $X$ is a null family if any of the following hold:

(i) Elements of $\mathcal{C}$ are pairwise disjoints connected open set of $X$.

(ii) Elements of $\mathcal{C}$ are the connected components of some subset $K$ of $X$.

Proof. The proof of (i) and (ii) are respectively in [8], Theorem 3 page 269 and Theorem 9 (iv) page 272 .

Lemma 2.8. Let $X$ be a hereditarily locally connected continuum and $F$ a proper closed subset of $X$. The quotient space induced by collapsing $F$ to a point is also a hereditarily locally connected continuum.

Proof. We denote by $\pi$ the natural quotient map. Let $A$ be a closed subset of $X$, then $\pi^{-1}(\pi(A))$ is closed, in fact, $\pi^{-1}(\pi(A))$ is either $A$ or $A \cup F$, therefore by [13], Proposition 3.7 and Theorem $3.10, \pi(X)$ is a continuum. Assume now that $\pi(X)$ is not hereditarily locally connected continuum, thus it has a convergence continuum. So we can find a sequence $\left(A_{n}\right)_{n \geq 0}$ in $C(\pi(X))$ converging to some $A \in C(\pi(X))$, such that for any $n \geq$ $0, A_{n} \cap A=\emptyset$ and $A$ is not degenerate.

We can assume that $\left(A_{n}\right)_{n \geq 0}$ is a pairwise disjoint family (by passing to a subsequence if needed), indeed let $A_{n_{0}}=A_{0}$ and $O_{1}$ an open neighbourhood of $A$ such that $A_{n_{0}} \cap O_{1}=\emptyset$. As $\left(A_{n}\right)_{n \geq 0}$ converges to $A$ we can find $n_{1}>0$ such that $A_{n_{1}} \subset O_{1}$.

Assume now that the family $\left(A_{n_{i}}\right)_{0 \leq i \leq N}$ has been defined such that $\left(A_{n_{i}}\right)_{0 \leq i \leq N}$ are pairwise disjoint. Let $O_{N+1}$ an open neighbourhood of $A$ such that

$$
\bigcup_{0 \leq i \leq N} A_{n_{i}} \cap O_{N+1}=\emptyset \text {. We can find } n_{N+1}>n_{N} \text { such that } A_{n_{N+1}} \subset O_{N+1} \text {, }
$$

thus $\left(A_{n_{i}}\right)_{0 \leq i \leq N+1}$ are pairwise disjoint. Continuing indicatively we get the subsequence $\left(A_{n_{i}}\right)_{i \geq 0}$ so that $\left(A_{n_{i}}\right)_{i \geq 0}$ are pairwise disjoints.

As $\left(A_{n_{i}}\right)_{i>0}$ are pairwise disjoints, we can assume that for any $i \geq 0$, $\pi(F) \notin A_{n_{i}}$. Therefore for any $i \geq 0, \pi^{-1}\left(A_{n_{i}}\right)$ is a subcontinuum of $X$, indeed the restriction of $\pi$ on $X \backslash F$ is an open continuous bijection, thus a homeomorphism ( Let $O$ be an open set of $X \backslash F$, then $\pi^{-1}(\pi(O))=O$ ). $C(X)$ is compact so we can assume that the sequence $\left(\pi^{-1}\left(A_{n_{i}}\right)\right)_{i \geq 0}$ converges to some $B \in C(X)$. The map $\pi$ is continuous and onto then:

$$
\pi(B)=\lim _{i \rightarrow+\infty} \pi\left(\pi^{-1}\left(A_{n_{i}}\right)\right)=\lim _{i \rightarrow+\infty} A_{n_{i}}=A .
$$

Thus $B \subset \pi^{-1}(A)$. Recall that for any $i \geq 0, \pi^{-1}\left(A_{n_{i}}\right) \cap \pi^{-1}(A)=\emptyset$, therefore $\pi^{-1}\left(A_{n_{i}}\right) \cap B=\emptyset$. As $X$ is a hereditarily locally connected continuum, then $B$ must be reduced to a point, therefore $\pi(B)=A$ is also reduced to a point which is a contradiction.

Lemma 2.9. Let $X$ be a continuum, the following assertions are equivalents:

(i) $X$ is hereditarily locally connected;

(ii) For any sequence $\left(A_{n}\right)_{n \geq 0}$ in $C(X)$ converging to $A \in C(X)$, we have 
$\lim _{n \rightarrow+\infty} \operatorname{Mesh}\left(A_{n} \backslash A\right)=0,($ where $\operatorname{Mesh}(B)=\sup \{\operatorname{diam}(C): C$ is a connected component of $B\}$ ).

(iii) For any sequence $\left(A_{n}\right)_{n \geq 0}$ of subcontinua of $X$ converging to a non degenerated subcontinuum $A$ of $X$, we have $\lim _{n \rightarrow+\infty} A_{n} \cap A=A$.

Proof. (i) $\Leftrightarrow$ (ii) The proof is Lemma 4.2 in [4].

(i) $\Rightarrow$ (iii): We can assume that $A$ is a proper subset of $X$ since otherwise there is nothing to prove. Let $L=\lim _{n \rightarrow+\infty} A_{n} \cap A$, by Lemma 2.9, since $A$ is non degenerated, $L \neq \emptyset$. Assume that $L \subsetneq A$ and let $x \in A \backslash L$. By (i) there exist $U$ an open neighborhood of $A$ such that $M e s h(U \backslash A)<\frac{d(L, x)}{4}$. We can assume that for any $n \geq 0, A_{n} \subset U$. Let $O_{L} \subset U$ be an open neighborhood of $L$ such that $d\left(x, \overline{O_{L}}\right)>\frac{d(L, x)}{2}$ and $x_{n} \in A_{n}$ so that $\left(x_{n}\right)_{n \geq 0}$ converges to $x$.

Let $n \geq 0$ large enough such that $A_{n} \cap A \subset O_{L}$ and $d\left(x_{n}, \overline{O_{L}}\right) \geq \frac{d(L, x)}{2}$. Recall that $A_{n}$ is arcwise connected so let $I$ be an arc of $A_{n}$ joining $x_{n}$ to a point of $A_{n} \cap A$ and let $J$ be the connected component of $I \backslash O_{L}$ that contains $x_{n}$. We have then $J \cap O_{L}=\emptyset$ and $J \cap \partial\left(O_{L}\right) \neq \emptyset$. Clearly $J \subset U \backslash A$, moreover $J$ is a connected subset of $X$ such that $\operatorname{diam}(J) \geq d\left(x_{n}, \partial\left(O_{L}\right)\right)>\frac{d(L, x)}{4}$, which is a contradiction.

(iii) $\Rightarrow$ (i) This follow immediately from the definition of a convergence continuum and the characterization of hereditarily locally connected continua given in Lemma 2.5

Similar to Lemma 2.9, we have also the following lemma:

Lemma 2.10. Let $X$ be a hereditarily locally connected continuum, $F \subsetneq$ $X$ a proper closed subset and $\left(O_{n}\right)_{n \geq 0}$ a sequence of open sets such that $F=\bigcap_{n \geq 0} \overline{O_{n}}$, then $\lim _{n \rightarrow+\infty} \operatorname{Mesh}\left(O_{n} \backslash F\right)=0$.

Proof. Let $F \subsetneq X$ a closed subset and $\left(O_{n}\right)_{n \geq 0}$ a sequence of open sets such that $F=\bigcap_{n \geq 0} \overline{O_{n}}$. Assume that $\limsup _{n \rightarrow+\infty} \operatorname{Mesh}\left(O_{n} \backslash F\right)>0$. Then $\lim _{i \rightarrow+\infty} \operatorname{Mesh}\left(O_{n_{i}} \backslash F\right)=\gamma>0$ for some increasing sequence $\left(n_{i}\right)_{i \geq 0}$ of positive integers. It follows that for some $k \in \mathbb{N}$ and for any $i \geq k$, there is a connected component $C_{i}$ of $O_{n_{i}} \backslash F$ with $\operatorname{diam}\left(C_{i}\right)>\frac{\gamma}{2}$. Recall that for any $i \in \mathbb{N}, O_{n_{i}}$ is a locally connected, thus $C_{i}$ is an arcwise connected open subset of $X$. For any $i \geq k$, let $J_{i}$ be an $\operatorname{arc}$ in $C_{i}$ with $\operatorname{diam}\left(J_{i}\right)>\frac{\gamma}{4}$. By compactness of $C(X)$, we may assume that $\left(J_{i}\right)_{i \geq k}$ converges to some $J \in C(X)$. We have $J \subset F$ and $\operatorname{diam}(J) \geq \frac{\gamma}{4}$, in fact as $\lim _{i \rightarrow+\infty} d_{H}\left(J_{i}, J\right)=0$, then any point $x \in J$ is a limit of a sequence $\left(x_{i}\right)_{i \geq 0}$, with $x_{i} \in J_{i} \subset O_{n_{i}}$, for any $i \geq k$. Moreover, for any $i \geq k$ we have $J_{i} \cap J \subset\left(O_{n_{i}} \backslash F\right) \cap F=\emptyset$. Hence $J$ is a convergence continuum which is a contradiction. 
The following two technical lemmas are essential and will be used throughout most of the proof of this paper:

Lemma 2.11. Let $X$ be a hereditarily locally connected continuum, $F$ a closed subset of $X, a \in F$ and $\left(a_{n}\right)_{n \geq 0}$ a sequence of $X \backslash F$ converging to $a$. If for some connected component $C$ of $X \backslash F$ and for any $n \geq 0, a_{n} \in C$. Then there exists a sequence of arc $\left(J_{n}\right)_{n \geq 0}$ with end points $a_{n}$ and $a$ converging to $\{a\}$ (with respect to the Hausdorrf metric) such that $J_{n} \cap F=\{a\}$, for infinitely many $n \geq 0$.

Proof. It will be enough to prove that for any neighbourhood $O$ of $a$ in $X$, there exists an arc $J \subset O$ satisfying the condition of the lemma.

First observe that $C$ is an open connected subset of $X$ thus arcwise connected. Let $V$ be a closed connected neighbourhood of $a$ such that $C \nsubseteq$ $V \subset O$, fix some $r \in C \backslash V$ and let $K_{n}$ be some arc of $C$ joining $a_{n}$ and $r$, clearly $K_{n} \cap \partial(V) \neq \emptyset$. Denote by $\epsilon=d(a, \partial(V))>0$, since $\left(a_{n}\right)_{n \geq 0}$ converges to $a$, there exists some $N \geq 0$ such that for any $n \geq N, a_{n} \in V$ and $d\left(a_{n}, \partial(V)\right)>\frac{\epsilon}{2}$. Recall that $\left(a_{n}\right)_{n>0}$ is a sequence of $X \backslash F$, so denote by $C_{n}$ the connected component of $V \backslash F$ containing $a_{n}$. Note that for each $n \geq N, C_{n} \cap \partial(V) \neq \emptyset$ (It will be enough to consider the subarc $K_{n}^{\prime} \subset V$ of $K_{n}$ joining $a_{n}$ to some point of $\left.\partial(V)\right)$. We conclude that for any $n \geq N$, $\operatorname{diam}\left(C_{n}\right)>\frac{\epsilon}{2}$. Therefore by Lemma $2.7\left\{C_{n}, n \geq N\right\}$ is finite, thus for some $n_{V} \geq N, C_{n}=C_{n_{V}}$, for infinitely many $n$, therefore $a \in C l_{V}\left(C_{n_{V}}\right)$, hence $a \in B d_{V}\left(C_{n_{V}}\right)$. Recall that $V$ is a hereditarily locally connected continuum and $C_{n_{V}}$ is an open connected set of $V$, by lemma $2.6 C_{n_{V}} \cup\{a\}$ is arcwise connected, so we let $J_{V} \subset C_{n_{V}} \cup\{a\}$ an arc joining $a$ and $a_{n_{V}}$. We conclude that $J_{V} \subset C_{n_{V}} \cup\{a\} \subset(V \backslash F) \cup\{a\} \subset O$, this ends the proof.

Before we give the second lemma we need some definitions and notations: Let $f: X \rightarrow X$ be a homeomorphism of a hereditarily locally connected continuum, $a \in F i x(f)$ and $O$ a connected open neighbourhood of $a$ in $X$. Denote by $\widetilde{I_{O}^{-}}=\left\{y \in \bar{O}, O_{f^{-}}(y) \subset \bar{O}\right\}$ and let $I_{O}^{-}$the connected component of $\widetilde{I_{O}^{-}}$containing $a$. Clearly $f^{-1}\left(I_{O}^{-}\right) \subset I_{O}^{-} \subset \bar{O}$ is a subcontinuum of $X$ and $f\left(X \backslash I_{O}^{-}\right) \subset X \backslash I_{O}^{-}$. Now we can state the lemma:

Lemma 2.12. Let $f: X \rightarrow X$ be a homeomorphism of a hereditarily locally connected continuum, $a \in F i x(f), O$ a connected open neighbourhood of $a$ and $\left(a_{n}\right)_{n \geq 0}$ a sequence of $O$ converging to $a$. Then the following assertions can not be simultaneously true:

(i) For any $n \geq 0$, there exists $m_{n} \geq 0$ such that $f^{m_{n}}\left(a_{n}\right) \notin O$;

(ii) There exist a sequence of continua $\left(J_{n}\right)_{n \geq 0}$ containing $a_{n}$ and $a$ such that for any $n \geq 0$ we have $J_{n} \cap I_{O}^{-}=\{a\}$;

(iii) The sequence $\left(J_{n}\right)_{n \geq 0}$ of (ii) also satisfy $\lim _{n \rightarrow+\infty} d_{H}\left(J_{n},\{a\}\right)=0$.

Proof. Assume that under the conditions of the lemma, assertion (i),(ii),(iii) are trues. By (iii), there exist $N \geq 0$ such that for any $n \geq N, J_{n} \subset O$ 
and with (i), for each $n \geq N$, we may find $s_{n} \geq 0$ such that for any $i \leq s_{n}, f^{i}\left(J_{n}\right) \subset O$ and $f^{s_{n}+1}\left(J_{n}\right) \nsubseteq O$.

Claim.1: The sequence $\left(s_{n}\right)_{n \geq N}$ is unbounded.

Indeed assume that this is not the case, then we may find a subsequence $\left(s_{m_{n}}\right)_{n \geq N}$ of $\left(s_{n}\right)_{n \geq N}$ and $k \geq 0$ such that $s_{m_{n}}=k$, for any $n \geq N$. By the definition of $\left(s_{n}\right)_{n \geq N}$, we get $f^{k+1}\left(J_{n}\right) \nsubseteq O$, recall that $a \in f^{k+1}\left(J_{n}\right)$, therefore for any $n \geq 0$ we have $f^{k+1}\left(J_{n}\right) \cap \partial(O) \neq \emptyset$. By continuity of $f^{k+1}$, the sequence $\left(f^{k+1}\left(J_{n}\right)\right)_{n>N}$ converges to $\{a\}$, hence $a \in \partial(O)$, which is a contradiction. This end the proof of the claim, we may assume then that $\left(s_{n}\right)_{n \geq N}$ is increasing and that the sequence $\left(f^{s_{n}}\left(J_{n}\right)\right)_{n \geq N}$ converges with respect to the Hausdroof metric, let then $J=\lim _{n \rightarrow+\infty} f^{s_{n}}\left(J_{n}\right)$.

Claim.2 $J$ is a non degenerated subcontinua of $X$, moreover $J \subset I_{O}^{-}$.

Clearly $J \in C(X)$, as proven above we have $f(J) \cap \partial(O) \neq \emptyset$ and $a \in$ $f(J) \cap O$, thus $J$ is non degenerated. For the rest since $a \in J$ and $J$ is connected, so it will be enough to prove that $J \subset \widetilde{I_{O}^{-}}$, equivalently for any $p \geq 0, f^{-p}(J) \subset \bar{O}$.

Let $p \geq 0$, we have $f^{-p}(J)=\lim _{n \rightarrow+\infty} f^{s_{n}-p}\left(J_{n}\right)$. By claim 1 , the sequence $\left(s_{n}\right)_{n \geq N}$ is increasing, thus there exists $N_{1} \geq N$ such that $s_{n}>p$, for any $n \geq N_{1}$, therefore by the definition of $\left(s_{n}\right)_{n \geq N}$ for any $n \geq N_{1}, f^{s_{n}-p}\left(J_{n}\right) \subset$ $\bar{O}$, therefore $f^{-p}(J) \subset \bar{O}$. This end the proof of claim 2 .

End of the proof of Lemma 2.12: By (iii) of Lemma 2.9, since $J=\lim _{n \rightarrow+\infty} f^{s_{n}}\left(J_{n}\right)$ and $J$ is non degenerated, then $J=\lim _{n \rightarrow+\infty} f^{s_{n}}\left(J_{n}\right) \cap J$, denote by $B_{n}=$ $f^{s_{n}}\left(J_{n}\right) \cap J$, clearly we may assume that for any $n \geq N, \operatorname{Card}\left(B_{n}\right)>1$. We have $f^{-s_{n}}\left(B_{n}\right)=J_{n} \cap f^{-s_{n}}(J)$, recall that by claim $2, J \subset I_{O}^{-}$, thus $f^{-s_{n}}(J) \subset I_{O}^{-}$. Therefore $f^{-s_{n}}\left(B_{n}\right) \subset J_{n} \cap I_{O}^{-}=\{a\}$, this contradict the fact that $\operatorname{Card}\left(B_{n}\right)>1$.

\section{3. limit sets and Nonwandering set for homeomorphism of hereditarily locally connected continua}

3.1. Limit sets for homeomorphism of hereditarily locally connected continua. The aim of this subsection is to extend theorem 2.1 in [14] and to prove the following theorem:

Theorem 3.1. Let $f: X \rightarrow X$ be a hereditarily locally connected continuum homeomorphism. For any $x \in X, \omega_{f}(x)$ is a minimal set.

Proof. Assume that for $x \in X, \omega_{f}(x)$ is not a minimal set, thus it contains a proper minimal subset $M$, by Lemma 2.8 , without lose of generality, me may collapse $M$ to a single point $\{a\}$ so that $\omega_{f}(x)$ is infinite and contains a fixed point $a$.

Claim.1: We can assume that $X \backslash\{a\}$ is connected.

Let $C$ be the connected component of $X \backslash\{a\}$ containing $x$, there must exist 
$n \geq 1$ such that $f^{n}(C)=C$, since if not $\left(f^{n}(C)\right)_{n \geq 0}$ are pairwise disjoints open connected subset of $X$. Thus by Lemma $2.7\left\{f^{n}(C), n \in \mathbb{N}\right\}$ is a null family, then so is the family $\left\{f^{n}(\bar{C}), n \in \mathbb{N}\right\}$. Hence $x$ and $a$ are asymptotic, which is a contradiction. So we can consider $X=\bar{C}$ and $g=f^{n}$ this end the proof of claim 1 .

$X \backslash\{a\}$ is an open connected set of $X$, thus arcwise connected. Let then $K$ be an arc joining $x$ and $f(x)$ such that $a \notin K$ and $O$ an open connected neighbourhood of $a$ in $X$ such that $K \cap \bar{O}=\emptyset$ and $\omega_{f}(x) \nsubseteq \bar{O}$.

Denote by $\widetilde{I^{-}}=\left\{y \in \bar{O}, O_{f^{-}}(y) \subset \bar{O}\right\}$ and let $I^{-}$the connected component of $\widetilde{I^{-}}$containing $a$. Clearly $f^{-1}\left(I^{-}\right) \subset I^{-}$is a closed subset of $X$ and $O_{f^{+}}(x) \cap I^{-}=\emptyset$ (since otherwise $x \in I^{-} \subset \bar{O}$ ).

Let $C_{x}$ be the connected component of $x$ of $X \backslash I^{-}$. We have $f^{-1}\left(I^{-}\right) \subset$ $I^{-}$, then $f\left(X \backslash I^{-}\right) \subset X \backslash I^{-}$, moreover $\{x, f(x)\} \subset K \subset X \backslash \overline{O_{0}} \subset X \backslash I^{-}$. Therefore $f\left(C_{x}\right) \subset C_{x}$, thus $O_{f^{+}}(x) \subset C_{x}$. Recall that $a \in \omega_{f}(x)$, so let $\left(s_{n}\right)_{n \geq 0}$ an increasing sequence of integers such that $\left(f^{s_{n}}(x)\right)_{n \geq 0}$ converges to $\{a\}$. Since for any $n \geq 0, f^{s_{n}}(x) \in C_{x}$, where $C_{x}$ is a connected component of $X \backslash I^{-}$, by Lemma 2.11, there exist a subsequence $\left(s_{m_{n}}\right)_{n \geq 0}$ of $\left(s_{n}\right)_{n \geq 0}$ and a sequence of arc $\left(I_{n}\right)_{n \geq 0}$ satisfying :

(i) $I_{n} \cap I^{-}=\{a\}$;

(ii) $\lim _{n \rightarrow+\infty} I_{n}=\{a\}$;

(iii) $f^{s_{m_{n}}}(x) \in I_{n}$.

Recall that $\omega_{f}(x) \nsubseteq \bar{O}$, then by (iii) for any $n \geq 0$ we can find $q_{n}>0$ such that $f^{q_{n}}\left(I_{n}\right) \not \subseteq O$. As conclusion we have:

(1) The sequence $\left(f^{s_{m_{n}}}(x)\right)_{n \geq 0}$ converges to $a$ such that for some sequence of positive integers $\left(q_{n}\right)_{n \geq 0}$, we have $f^{q_{n}}\left(f^{s_{m_{n}}}(x)\right) \notin O$;

(2) There exist a sequence of $\operatorname{arcs}\left(I_{n}\right)_{n \geq 0}$ joining $\left(f^{s_{m_{n}}}(x)\right)$ and $a$ such that for any $n \geq 0$ we have $I_{n} \cap I^{-}=\{a\}$;

(3) $\lim _{m \rightarrow+\infty} \bar{d}_{H}\left(I_{n},\{a\}\right)=0$.

Therefore the three assertions of Lemma 2.12 are trues, this will lead to a contradiction. Therefore $\omega_{f}(x)$ is a minimal set.

Corollary 3.2. Let $f: X \rightarrow X$ be a hereditarily locally connected continuum homeomorphism, the following hold:

(i) For any $x \in X, \alpha_{f}(x)$ is a minimal set;

(ii) $A P(f)=R(f)=\Lambda(f)$.

3.2. Nonwandering sets of homeomorphism of hereditarily locally connected continuum. The aim of this section is to prove the following theorem which extends theorem 2.1 of [15].

Theorem 3.3. Let $f: X \rightarrow X$ be a hereditarily locally connected continuum homeomorphism. Then $\Omega(f)=\Lambda(f)=R(f)=A P(f)$.

Proof. By (ii) of Corollary 3.2, it will be enough to prove that $\Omega(f) \subset R(f)$. Let $x \in \Omega(f) \backslash R(f)$, thus $x \notin \omega_{f}(x)$, we can assume by Lemma 2.8 that 
$\omega_{f}(x)$ is reduced to a fixed point $a$.

Let $O$ an open connected neighborhood of $a$ such that $x \notin \bar{O}, \widetilde{I}=\{y \in$ $\left.\bar{O}, O_{f^{-1}}(y) \subset \bar{O}\right\}$ and $I$ the connected component of $\widetilde{I}$ containing a, clearly $f^{-1}(I) \subset I$ and $O_{f^{+}}(x) \cap I=\emptyset$. Let then $C_{x}$ be the connected component of $X \backslash I$ that contains $x$. Since $x \in \Omega(f)$, we may find $\left(x_{k}\right)_{k \geq 0}$ a sequence in $X$ and $\left(n_{k}\right)_{k \geq 0}$ an increasing sequence of integers such that the sequences $\left(x_{k}\right)_{k \geq 0}$ and $\left(f^{n_{k}}\left(x_{k}\right)\right)_{k \geq 0}$ converges to $x$.

Claim: For $k$ large enough, $f^{n_{k}}(x) \in C_{x}$.

$X$ is a locally connected continuum, then $C_{x}$ the connected component of $X \backslash I$ containing $x$ is an open neighbourhood of $x$ in $X$. Since $\left(x_{k}\right)_{k \geq 0}$ and $\left(f^{n_{k}}\left(x_{k}\right)\right)_{k \geq 0}$ converges to $x$, then for $k$ large enough, $\left\{x_{k}, f^{n_{k}}\left(x_{k}\right)\right\} \subset C_{x}$. The sequence $\left(x_{k}\right)_{k \geq 0}$ converges to $x$, so for $k$ large enough, we can find a sequence $J_{k}$ of arc joining $x$ and $x_{k}$ such that $J_{k} \cap I=\emptyset$, thus $f^{n_{k}}\left(J_{k}\right) \cap I=\emptyset$, moreover $f^{n_{k}}\left(J_{k}\right)$ joins $f^{n_{k}}(x)$ and $f^{n_{k}}\left(x_{k}\right)$ where $f^{n_{k}}\left(x_{k}\right) \in C_{x}$, therefore $f^{n_{k}}(x) \in f^{n_{k}}\left(J_{k}\right) \subset C_{x}$. This end the proof of the claim.

Recall that $\omega_{f}(x)=\{a\}$, therefore $\left(f^{n_{k}}(x)\right)_{k \geq 0}$ converges to $\{a\}$, moreover by the claim above, for $k$ large enough, $f^{n_{k}}(x) \in C_{x}$, where $C_{x}$ is a connected component of $X \backslash I$. By Lemma 2.11, we may find a sequence of arc $\left(L_{k}\right)_{k>0}$ satisfying the follow :

(i) $L_{k} \cap I=\{a\}$;

(ii) $\lim _{k \rightarrow+\infty} L_{k}=\{a\}$;

(iii) $f^{n_{k}}(x) \in L_{k}$;

Let $s \geq 0$, recall that the sequence $\left(x_{k}\right)_{k \geq 0}$ converges to $x$, then $\left(f^{n_{s}}\left(x_{k}\right)\right)_{k \geq 0}$ converges to $f^{n_{s}}(x)$. Since $O_{f^{+}}(x) \cap I=\emptyset$, let $V_{s}$ some arcwise connected open neighbourhood of $f^{n_{s}}(x)$, thus for $k$ large enough, $f^{n_{s}}\left(x_{k}\right) \in V_{s}$. The sequence of integers $\left(n_{k}\right)_{k \geq 0}$ is increasing, therefore we may find some $k_{s} \geq 0$ such that $f^{n_{s}}\left(x_{k_{s}}\right) \in V_{s}$ and $n_{k_{s}}>n_{s}$. For any $s \geq 0$, we let then $K_{s}$ some arc in $V_{s}$ joining $f^{n_{s}}(x)$ and $f^{n_{s}}\left(x_{k_{s}}\right)$, so that the family $\left\{K_{s}, s \geq 0\right\}$ is a null family.

Let $I_{s}=L_{s} \cup K_{s}$, clearly $\left(I_{s}\right)_{s \geq 0}$ is a family of subcontinua of $X$ satisfying the follow :

(i) $I_{s} \cap I=\{a\}$;

(ii) $\lim _{s \rightarrow+\infty} I_{s}=\{a\}$;

(iii) $f^{n_{s}}\left(x_{k_{s}}\right) \in I_{s}$ and $n_{k_{s}}>n_{s}$.

By (iii) for any $s \geq 0$ we can find $q_{s}>0,\left(q_{s}=n_{k_{s}}-n_{s}\right)$ such that $f^{q_{s}}\left(I_{s}\right) \nsubseteq \bar{O}$ (in fact $f^{n_{s_{k}}}\left(x_{k_{s}}\right)$ converges to $\left.x \in X \backslash \bar{O}\right)$. This will contradict Lemma 2.12, thus $\Omega(f) \subset R(f)$ and the result follow.

\section{Continuity of the maps: $\omega_{f}$ AND $\alpha_{f}$}

Let $X$ be a hereditarily locally connected continuum and $f$ a self homeomorphism of $X$. For $x \in X$ we define the maps : $\omega_{f}: X \rightarrow 2^{X}$ respectively 
$\alpha_{f}: X \rightarrow 2^{X}$, sending $x$ to its $\omega_{f}(x)$ respectively to its $\alpha_{f}(x)$. The main purpose of this section is to prove Theorems 4.1, 4.3, among other lemmas that will be used in the next sections.

Theorem 4.1. Let $f: X \rightarrow X$ be a hereditarily locally connected continuum homeomorphism and $\left(M_{n}\right)_{n \geq 0}$ a sequence of minimal set converging to some $M \subset X$, then $M$ is also a minimal set.

Proof. Let $\left(M_{n}\right)_{n \geq 0}$ a sequence of minimal sets converging to $M$ and assume that $M$ is not minimal. By Theorem 3.3, $R(f)$ is a closed set thus $M \subset R(f)$, moreover $f(M)=M$, therefore by Theorem 3.1, $M=\bigcup_{i \in I}^{\emptyset} \omega_{f}\left(x_{i}\right)$, where $\operatorname{Card}(I)>1$. By Lemma 2.8, we may collapse two minimal set contained in $M$ so that we can assume that $M$ contains two fixed points $a \neq b$.

Let then $O_{a}$ be an open connected neighbourhood of $a$ such that $b \notin \overline{O_{a}}$. Let $I^{-}$be the connected component of $\widetilde{I_{O_{a}}^{-}}$containing $a$. Since for any $n \geq 0, M_{n} \nsubseteq \overline{O_{a}}$, then for any $n \geq 0, M_{n} \cap I=\emptyset$, moreover $b \notin I$. So let $C_{b}$ be the connected component of $X \backslash I$ containing $b$. Observe that $f\left(C_{b}\right) \subset C_{b}$ is an open set of $X$ and for any $n \geq 0, M_{n} \cap C_{b} \neq \emptyset$, thus by minimality of $\left(M_{n}\right)_{n \geq 0}$, we get $M_{n} \subset C_{b}$, for any $n \geq 0$.

Recall that $a \in \lim _{n \rightarrow+\infty} M_{n}$, so for each $n \geq 0$, me way pick $a_{n} \in M_{n}$ so that the sequence $\left(a_{n}\right)_{n \geq 0}$ converges to $a$. For any $n \geq 0, a_{n} \in M_{n} \subset C_{b}$ a connected component of $X \backslash I$, then by Lemma 2.11 , we may find a sequence $\left(J_{n}\right)_{n \geq 0}$ of arc satisfying:

(i) $J_{n} \cap I=\{a\}$;

(ii) $\lim _{n \rightarrow+\infty} J_{n}=\{a\}$;

(iii) $a_{n} \in J_{n}$.

By (iii) since $a_{n} \in M_{n}$, where $M_{n}$ is a minimal set and $M_{n} \nsubseteq \bar{O}$, we can find some $q_{n}>0$ such that $f^{q_{n}}\left(a_{n}\right) \notin \bar{O}$, this will contradict Lemma 2.12, we conclude that $M$ is a minimal set.

Corollary 4.2. Let $f: X \rightarrow X$ be a hereditarily locally connected continuum homeomorphism, then the maps $\omega_{f}: \Omega(f) \rightarrow 2^{X}$ and $\alpha_{f}: \Omega(f) \rightarrow 2^{X}$ are continuous.

Proof. Let $\left(x_{n}\right)_{n \geq 0}$ a sequence of $\Omega(f)$ converging to some $x \in \Omega(f)$. By Theorem 3.3, $x_{n} \in \omega_{f}\left(x_{n}\right)$ and by Theorem 3.1, $\left(\omega_{f}\left(x_{n}\right)\right)_{n \geq 0}$ is a sequence of minimal set, thus by Theorem 4.1, any limit point of that sequence should be a minimal set. Observe that $x$ belongs to any limit point of the sequence $\left(\omega_{f}\left(x_{n}\right)\right)_{n \geq 0}$, therefore any limit point of the sequence $\left(\omega_{f}\left(x_{n}\right)\right)_{n \geq 0}$ is a minimal set that contains $x$, therefore it has to be $\omega_{f}(x)$.

By the same argument, we prove the continuity of the map $\alpha_{f}$.

Theorem 4.3. Let $f: X \rightarrow X$ be a hereditarily locally connected continuum homeomorphism. The maps $\omega_{f}: X \rightarrow 2^{X}$ and $\alpha_{f}: X \rightarrow 2^{X}$ are continuous everywhere except may be at the periodic point of $f$.

For the proof we need the following lemmas: 
Lemma 4.4. Let $f: X \rightarrow X$ be a hereditarily locally connected continuum homeomorphism and $x \in X$. If $\left(f^{n}(x), f^{m}(x)\right) \in P(X, f)$, for some $n<$ $m \in \mathbb{Z}$, then $\omega_{f}(x)$ is a periodic orbit.

Proof. Let $x \in X$ and suppose that $\left(f^{n}(x), f^{m}(x)\right) \in P(X, f)$ for some integers $n<m$. Then there exists a sequence of positive integers $\left(n_{i}\right)_{i \geq 0}$ such that $\lim _{i \rightarrow+\infty} d\left(f^{n_{i}}\left(f^{n}(x)\right), f^{n_{i}}\left(f^{m}(x)\right)\right)=\lim _{i \rightarrow+\infty} d\left(f^{n_{i}+n}(x), f^{m-n}\left(f^{n_{i}+n}(x)\right)\right)=$ 0 . Since $X$ is compact, we can assume that $\left(f^{n_{i}+n}(x)\right)_{i \geq 0}$ converges to some point $c \in X$. By continuity of $f^{m-n}$ We get $f^{m-n}(c)=c$. Therefore $c \in P(f) \cap \omega_{f}(x)$, by Theorem 3.1, $\omega_{f}(x)=O_{f}(c)$.

Lemma 4.5. Let $f: X \rightarrow X$ be a hereditarily locally connected continuum homeomorphism and $x \notin \Omega(f)$. Denote by $C_{x}$ the connected component of $x$ in $X \backslash \Omega(f)$, then the following hold :

(i) $C_{x} \times C_{x} \subset A(X, f) \cap A\left(X, f^{-1}\right)$,

(ii) If $\omega_{f}(x)$ is infinite, then $\overline{C_{x}} \times \overline{C_{x}} \subset A(X, f) \cap A\left(X, f^{-1}\right)$;

(iii) There exist $z_{1} \in \partial\left(C_{x}\right) \cap \omega_{f}(x)$ and $z_{2} \in \partial\left(C_{x}\right) \cap \alpha_{f}(x)$ such that for any $y \in C_{x}$ we have $\left(z_{1}, y\right) \in A(X, f)$ and $\left(z_{2}, y\right) \in A\left(X, f^{-1}\right)$.

Proof. (i) Denote by $A$ the set of points in $C_{x}$ asymptotic to $x$ with respect to $f$ and $f^{-1}$. Clearly, $A$ is not empty. We will show that it is a clopen subset of $C_{x}$. Recall that $C_{x} \cap \Omega(f)=\emptyset$, thus any point $y \in A$ is wandering thus it admits a wandering neighborhood $U$. As $X$ is hereditarily locally connected continuum, we may choose $U$ to be connected. By Lemma 2.7, $\lim _{|n| \rightarrow+\infty} \operatorname{diam}\left(f^{n}(U)\right)=0$. It turn out that $U$ is a part from the asymptotic class of $y$ with respect to $f$ and $f^{-1}$. Therefore $A$ is open .

Now let $\left(y_{n}\right)_{n \geq 0}$ be a sequence in $A$ converging to a point $y$ in $C_{x}$. Similarly as above, we can find an open wandering connected neighborhood $O$ of $y$ in $X$, so $\lim _{|n| \rightarrow+\infty} \operatorname{diam}\left(f^{n}(O)\right)=0$. For some $N \in \mathbb{N}, y_{N} \in O$ and then $\left(y_{N}, y\right)$ is an asymptotic pair with respect to $f$ and $f^{-1}$ and so does $(x, y)$ which implies that $y \in A$. Thus $A$ is also a closed subset of $C_{x}$. It turn out that $A$ is a clopen non empty subset of $C_{x}$, since $C_{x}$ is connected we conclude that $A=C_{x}$.

(ii) Observe that the sequence $\left(f^{n}\left(C_{x}\right)\right)_{n \in \mathbb{Z}}$ is pairwise disjoint, since otherwise if $f^{n}\left(C_{x}\right) \cap f^{m}\left(C_{x}\right) \neq \emptyset$, for some $n<m \in \mathbb{Z}$, then by (i) $x$ and $f^{m-n}(x)$ are asymptotic with respect to $f$, thus by Lemma $4.4, \omega_{f}(x)$ will be finite which is a contradiction. Therefore by Lemma $2.7\left(f^{n}\left(C_{x}\right)\right)_{n \in \mathbb{Z}}$ is a null family, thus the result follow.

(iii) Observe that $\partial\left(C_{x}\right) \subset \Omega(f)$, moreover if $y \in \Omega(f)$ and $x \in X$ such that $(x, y) \in P(X, f)$, then $y \in \omega_{f}(x)$. Indeed, in the first hand by Theorem 3.1 , any $\omega$-limit set is minimal, thus $\omega_{f}(x)=\omega_{f}(y)$, in the second hand, by Theorem 3.3, we have $y \in \omega_{f}(y)$, therefore $y \in \omega_{f}(x)$. So to prove (iii) it will be enough to prove that there exist $z_{1} \in \partial\left(C_{x}\right)$ and $z_{2} \in \partial\left(C_{x}\right)$ such that for any $y \in C_{x}$ we have $\left(z_{1}, y\right) \in A(X, f)$ and $\left(z_{2}, y\right) \in A\left(X, f^{-1}\right)$. If $\omega_{f}(x)$ is infinite, then the result follow from (ii). Assume now that $\omega_{f}(x)$ is finite, thus $\omega_{f}(x)=O_{f}(t)$, where $t \in P(f)$. By (i), for any 
$y \in C_{x}, \omega_{f}(y)=O_{f}(t)$ is finite. We distinguish two cases:

Case 1. $\left(f^{n}\left(C_{x}\right)\right)_{n \in \mathbb{Z}}$ is pair wise disjoint.

By Lemma 2.7, $\left(f^{n}\left(C_{x}\right)\right)_{n \in \mathbb{Z}}$ is a null family, thus $\overline{C_{x}} \times \overline{C_{x}} \subset A(X, f)$. Recall that $\partial\left(C_{x}\right) \subset \Omega(f)$, so let $z \in \partial\left(C_{x}\right)$, thus $(z, y) \in A(X, f) \cap A\left(X, f^{-1}\right)$, for any $y \in C_{x}$, we let then $z_{1}=z_{2}=z$.

Case 2. For some integers $n<m, f^{n}\left(C_{x}\right) \cap f^{m}\left(C_{x}\right) \neq \emptyset$.

In this case, we have $f^{p}\left(\overline{C_{x}}\right)=\overline{C_{x}}$, where $p=m-n$. Hence $O_{f}(t) \cap \partial\left(C_{x}\right) \neq$ $\emptyset$. Moreover for any $y \in C_{x}$, we have $\omega_{f} p(y) \subset O_{f}(t)$, therefore for any $y \in$ $C_{x}$, there exist $t_{y} \in O_{f}(t) \cap \partial\left(C_{x}\right)$ such that $\left(y, t_{y}\right) \in A\left(X, f^{p}\right) \subset A(X, f)$. By (i) we have $C_{x} \times C_{x} \subset A(X, f)$, hence for any $\left(y, y^{\prime}\right) \in C_{x} \times C_{x},\left(t_{y}, t_{y^{\prime}}\right) \in$ $A(X, f)$. It turn out that $t_{y}=t_{y^{\prime}}=t_{x}$, since any pair of periodic point is either equal or distal, we let then $z_{1}=t_{x}$.

The exitance of $z_{2}$ is proven in the same way, by replacing $f$ with $f^{-1}$.

Lemma 4.6. Let $f: X \rightarrow X$ be a hereditarily locally connected continuum homeomorphism and $x \in X$. If $\omega_{f}(x)$ or $\alpha_{f}(x)$ is infinite, then $\omega_{f}(x)=$ $\alpha_{f}(x)$.

Proof. If $x \in \Omega(f)=R(f)$, the result follow by minimality (see Theorem 3.1 and (i), Corollary 3.2). So we can assume that $x \notin \Omega(f)$, so let $C_{x}$ the connected component of $X \backslash \Omega(f)$ containing $x$. By (ii) of Lemma 4.5, $\overline{C_{x}} \times \overline{C_{x}} \subset A(X, f) \cap A\left(X, f^{-1}\right)$. Thus there exist $x^{\prime} \in \Omega(f) \cap \overline{C_{x}}$ such that $\left(x, x^{\prime}\right) \in A(X, f) \cap A\left(X, f^{-1}\right)$, thus by minimality any $\omega_{f}\left(\right.$ resp. $\left.\alpha_{f}\right)$-limit set we get, $\omega_{f}(x)=\omega_{f}\left(x^{\prime}\right)=\alpha_{f}\left(x^{\prime}\right)=\alpha_{f}(x)$.

Lemma 4.7. Let $f: X \rightarrow X$ be a hereditarily locally connected continuum homeomorphism and $x \in X$, for any sequence $\left(x_{n}\right)_{n>0}$ converging to $x$ at least one of the sequence $\left(\omega_{f}\left(x_{n}\right)\right)_{n \geq 0}$ or $\left(\alpha_{f}\left(x_{n}\right)\right)_{n \geq 0}$ converges to $\omega_{f}(x)$.

Proof. Let $M=\omega_{f}(x)$ and $L_{+}$(resp. $\left.L_{-}\right)$be the limit of $\left(\omega_{f}\left(x_{n}\right)\right)_{n \geq 0}$ (resp. $\left.\left(\alpha_{f}\left(x_{n}\right)\right)_{n \geq 0}\right)$. Denote by $L=L_{+} \cup L_{-}$, by Theorem $4.1, L_{+}$and $L_{-}$are minimal sets, assume that $M \neq L_{+}$and $M \neq L_{-}$, then $M \cap L=\emptyset$. By Lemma 2.8, we may assume that $M$ (resp. $L_{-}$) are reduced to fixed points $a$ (resp. $l_{-}$). Let $O_{a}$ be an open connected neighbourhood of $a$ such that $L \cap \overline{O_{a}}=\emptyset$ and $I^{-}$be the connected component of $\widetilde{I_{O_{a}}^{-}}$containing $a$. Since $\left(x_{n}\right)_{n \geq 0}$ converge to $x$ and $\omega_{f}(x)=\{a\}$, we may find an increasing sequence of positive integer $\left(m_{n}\right)_{n \geq 0}$ such that for any $n \geq 0, f^{m_{n}}\left(x_{n}\right) \in O_{a}$ and $\left(f^{m_{n}}\left(x_{n}\right)\right)_{n \geq 0}$ converges to $a$. Clearly $l_{-} \notin I^{-}$, so let $C_{l_{-}}$be the connected component of $X \backslash I^{-}$that contains $l_{-}$. Since $X \backslash I^{-}$is an $f$-invariant open set of $X$ and $l_{-}$is fixed point, then $f\left(C_{l_{-}}\right) \subset C_{l_{-}}$, moreover it contains $\alpha_{f}\left(x_{n}\right)$ eventually $\left(\left(\alpha_{f}\left(x_{n}\right)\right)_{n \geq 0}\right.$ converges to $\left.\left\{l_{-}\right\}\right)$. Therefore for $n$ large enough, $O_{f^{-1}}\left(x_{n}\right) \cap C_{l_{-}} \neq \emptyset$, hence for some $N \geq 0$, the sequence $\left(f^{m_{n}}\left(x_{n}\right)\right)_{n \geq N}$ is a sequence of $C_{l_{-}}$a connected component of $X \backslash I^{-}$such that $\left(f^{m_{n}}\left(x_{n}\right)\right)_{n \geq N}$ converges to $a \in I^{-}$, therefore by Lemma 2.11, we may find a sequence of $\operatorname{arc}\left(J_{n}\right)_{n \geq N}$ satisfying:

(i) $J_{n} \cap I^{-}=\{a\}$;

(ii) $\lim _{n \rightarrow+\infty} J_{n}=\{a\}$; 
(iii) $f^{m_{n}}\left(x_{n}\right) \in J_{n}$.

Recall that $\omega_{f}\left(x_{n}\right)$ converges to $L_{+}$, moreover $L_{+} \cap \overline{O_{a}}=\emptyset$, thus for $n$ large enough we have $\omega_{f}\left(x_{n}\right) \cap \overline{O_{a}}=\emptyset$, hence there exist $N_{0} \geq N$ such that for any $n \geq N_{0}$ there exist $q_{n} \geq 0$ such that $f^{q_{n}}\left(f^{m_{n}}\left(x_{n}\right)\right) \notin \overline{O_{a}}$, thus by (iii) $f^{q_{n}}\left(J_{n}\right) \nsubseteq \overline{O_{a}}$. This will contradict Lemma 2.12 .

Proof of Theorem 4.3: Let $x \in X \backslash P(f)$ and $\left(x_{n}\right)_{n \geq 0}$ a sequence of $X$ converging to $x$ such that $\left(\omega_{f}\left(x_{n}\right)\right)_{n \geq 0}$ converges to $L \neq \omega_{f}(x)$. By (i) of Lemma 4.5, $x \in \Omega(f)$ (since if not then $\left(x, x_{n}\right) \in A(X, f)$ for $n$ large enough), thus by Corollary 4.2, for $n$ large enough $x_{n} \notin \Omega(f)$ (if not then $\left(\omega_{f}\left(x_{n}\right)\right)_{n \geq 0}$ converges to $\left.\omega_{f}(x)\right)$, we can assume then that for any $n \geq 0, x_{n} \notin \Omega(f)$.

So let $C_{n}$ be the connected component of $X \backslash \Omega(f)$ that contains $x_{n}$. By Lemma 4.5 (iii), for each $n \geq 0$, there exist $z_{n} \in \Omega(f) \cap \partial\left(C_{n}\right)$ such that $\left(x_{n}, z_{n}\right) \in A(X, f)$, therefore for any $n \geq 0, \omega_{f}\left(x_{n}\right)=\omega_{f}\left(z_{n}\right)$.

Claim The family $\mathcal{C}=\left\{C_{n}, n \geq 0\right\}$ is finite.

Assume this is not the case a denote by $\mathcal{C}_{1}=\left\{C_{n_{i}}, i \geq 0\right\}$ an infinite subfamily of $\mathcal{C}$ such that the element of $\mathcal{C}$, are pairwise disjoint, hence the family $\mathcal{C}$, is null, therefore $\lim _{i \rightarrow+\infty} d\left(z_{n_{i}}, x_{n_{i}}\right)=0$. Thus the sequence $\left(z_{n_{i}}\right)_{i \geq 0}$ is a sequence of $\Omega(f)$ converging to $x$, by Corollary 4.2 , the sequence $\left(\omega_{f}\left(z_{n_{i}}\right)\right)_{i \geq 0}$ converges to $\omega_{f}(x)$, therefore $L=\omega_{f}(x)$, a contradiction. This ends the proof of the claim.

End of the proof of Theorem 4.3: By the Claim $\mathcal{C}=\left\{C_{n}, n \geq 0\right\}$ is finite, thus we can assume that for any $n \geq 0, x_{n} \in C_{0}$. By Lemma 4.5, (i), we have $C_{0} \times C_{0} \subset A(X, f) \cap A\left(X, f^{-1}\right)$, hence for any $n \geq 0, \omega_{f}\left(x_{n}\right)=\omega_{f}\left(x_{0}\right)$ and $\alpha_{f}\left(x_{n}\right)=\alpha_{f}\left(x_{0}\right)$. By Lemma 4.7, either $\left(\omega_{f}\left(x_{n}\right)\right)_{n \geq 0}$ converges to $\omega_{f}(x)$ or $\left(\alpha_{f}\left(x_{n}\right)\right)_{n \geq 0}$ converges to $\omega_{f}(x)$. Therefore since $\left(\omega_{f}\left(x_{n}\right)\right)_{n \geq 0}$ converges to $L \neq \omega_{f}(x), \quad\left(\alpha_{f}\left(x_{n}\right)\right)_{n \geq 0}$ must converges to $\omega_{f}(x)$, hence for any $n \geq$ $0, \alpha_{f}\left(x_{n}\right)=\omega_{f}(x)$, recall that $x \in \Omega(f) \backslash P(f)$, thus $\omega_{f}(x)$ is infinite, then by Lemma 4.6, we get for any $n \geq 0, \omega_{f}\left(x_{n}\right)=\alpha_{f}\left(x_{n}\right)=\omega_{f}(x)$, thus will imply that $L=\omega_{f}(x)$ which is a contradiction.

\section{The basin of attraction and periodic points of hereditarily locally connected continuum homeomorphism}

5.1. Basin of attraction for hereditarily locally connected continuum homeomorphism. Let $X$ be a hereditarily locally connected continuum, $f$ a self homeomorphism of $X$. If $M$ is a minimal set of $f$, we call $A(M)=\left\{x \in X: \omega_{f}(x)=M\right\}$ the basin of attraction of $M$. Clearly for any minimal set, $f(A(M))=A(M)$.

The aim of this subsection is to prove Theorem 5.1, this and the results of the first two sections will enable us to prove the density of the set of periodic point in the set of non-wandering point (Theorem 5.3). 
Theorem 5.1. Let $f: X \rightarrow X$ be a hereditarily locally connected continuum homeomorphism. If $M$ is an infinite minimal set of $f$, then $A(M)$ is a closed in $X$.

Proof. Under the conditions of Theorem 5.1, let $M$ be an infinite minimal set and $\left(x_{n}\right)_{n \geq 0}$ a sequence of $A(M)$ converging to $x$. Since $M$ is infinite then by Lemma 4.6, for any $n \geq 0, \omega_{f}\left(x_{n}\right)=\alpha_{f}\left(x_{n}\right)=M$. By Lemma 4.7 , one of the sequences $\left(\omega_{f}\left(x_{n}\right)\right)_{n \geq 0}$ or $\left(\alpha_{f}\left(x_{n}\right)\right)_{n \geq 0}$ converges to $\omega_{f}(x)$, therefore $\omega_{f}(x)=M$.

Note that if $M$ is finite, it may happened that $A(M)$ is not closed, as example consider the unit closed interval $t \rightarrow \sqrt{t}, A(\{1\})=] 0,1]$, which is not closed. Observe that in this case the $\overline{A(\{1\})} \backslash A(\{1\})=\{0\}$, which is a periodic point. With Theorem 4.3, we may prove that if a limit point of $A(M)$ does not belong to $A(M)$, then it has to be a periodic point.

Proposition 5.2. Let $f: X \rightarrow X$ be a hereditarily locally connected continuum homeomorphism. If for some finite minimal set $M, A(M)$ is not closed. Then $\overline{A(M)} \backslash A(M) \subset P(f)$

Proof. Let $y \in \overline{A(M)}$ and $\left(y_{n}\right)_{n \geq 0}$ a sequence of $A(M)$ converging to $y$. Assume that $y \notin P(f)$, by Theorem 4.3, the map $\omega_{f}$ is continuous at $y$. Thus $\left(\omega_{f}\left(y_{n}\right)\right)_{n \geq 0}$ converges to $\omega_{f}(y)$. Recall that for any $n \geq 0, \omega_{f}\left(y_{n}\right)=M$, therefore $\omega_{f}(y)=M$ and $y \in A(M)$.

\subsection{On infinite minimal sets and periodic points.}

Theorem 5.3. Let $f: X \rightarrow X$ be a hereditarily locally connected continuum homeomorphism. If $P(f) \neq \emptyset$, then $\overline{P(f)}=\Omega(f)$.

Before we prove Theorem 5.3, we need to establish first some results and recall the definition of local separating point in the case of hereditarily locally connected continuum.

Let $X$ be a hereditarily locally connected continua and $x \in X$, the point $x$ is said to be a local separating point of $X$, if there exist a connected neighborhood $O_{x}$ of $x$ such that $O_{x} \backslash\{x\}$ is not connected. We denote by $L(X)$ the set of local separating point $X$.

Note that since a hereditarily locally connected continuum is locally connected, this definition is equivalent to the one of local separating point given by Whyburn in [21]. (see (9.5) in [22],). This lemma stat some properties of local separating point which will be used in the proof of Lemma 5.6.

Lemma 5.4. Let $X$ be a continuum, then the following hold:

(i) If $C$ a connected subset of $X$, then $(\bar{C} \backslash C) \cap L(X)$ is a most countable.

(ii) If $X$ is a rational continuum, in particular a hereditarily locally connected continuum then $L(X)$ is a dense subset of $X$.

Proof. For the proof of (i) see [21] and for the proof of (ii) see (9.43) in [22] 
Given a hereditarily locally connected continuum, we denote by $\mathcal{M}$ (resp. $\mathcal{M}_{\infty}$ ) the set of minimal set (resp. infinite minimal set) of $f$ and by $\mathcal{M}_{A F}$, the set of minimal set $M$, for which $A(M)$ has a finite number of connected component.

Lemma 5.5. Let $f: X \rightarrow X$ be a hereditarily locally connected continuum homeomorphism and $M$ some minimal set. For any connected component $C$ of $A(M)$, we have $C \cap M \neq \emptyset$.

Proof. It will be enough to prove that for any $x \in A(M) \backslash M$, the connected component of $x$ in $A(M)$ meets $M$. By Theorem 3.3, if $x \in A(M) \backslash M$ then $x \notin \Omega(f)$. So let $C_{x}$ be the connected component of $X \backslash \Omega(f)$ containing $x$. By (iii) of Lemma 4.5, we may find some $z \in \omega_{f}(x) \cap \partial\left(C_{x}\right)$ such that $(y, z) \in A(X, f)$, for any $y \in C_{x}$. Therefore $\left(C_{x} \cup\{z\}\right) \times\left(C_{x} \cup\{z\}\right) \subset A(X, f)$. Thus $C_{x} \cup\{z\} \subset A\left(\omega_{f}(x)\right)=A(M)$, moreover $\left(C_{x} \cup\{z\}\right)$ is a connected subset of $X$. Thus the result follow.

By Lemma 5.5, for any $M \in \mathcal{M}_{A F}$, the connected component $\left(C_{i}\right)_{0 \leq i \leq n_{M}}$ of $A(M)$ form a periodic cycle of length $n_{M}$. Observe also that any periodic orbit is an element of $\mathcal{M}_{A F}$.

Recall that a periodic cycle with length $n$ is a family $F_{0}, F_{1}, \ldots F_{n-1}$ of $n$ subsets of $X$, where $f\left(F_{i}\right)=F_{i+1}$ and $f\left(F_{n-1}\right)=F_{0}$. In the case where $F_{0}$ is closed, it will be a periodic point for $2^{f}$. We denote by $O P_{f}\left(F_{0}\right)=$ $\bigcup f^{i}\left(F_{0}\right) \in 2^{X}$.

$0 \leq i \leq n$

Let $\mathcal{M}_{A F}(X)=\bigcup_{M \in \mathcal{M}_{A F}} M \subset \Omega(f)$, the first step of the proof of Theorem 5.3 , is the following lemma.

Lemma 5.6. Let $f: X \rightarrow X$ be a hereditarily locally connected continuum homeomorphism, then $\Omega(f)=\overline{\mathcal{M}_{A F}(X)}$.

Proof. Assume that $\overline{\mathcal{M}_{A F}(X)} \subsetneq \Omega(f)$. Clearly, $\overline{\mathcal{M}_{A F}(X)}$ is a closed strongly $f$-invariant subset of $X$, moreover by Theorem 3.3, $\Omega(f)=A P(f)$, then there exists some minimal set $N$ such that $N \cap \overline{\mathcal{M}_{A F}(X)}=\emptyset$, so denote by $\mathcal{N}=\left\{L \in \mathcal{M} ; L \cap \overline{\mathcal{M}_{A F}(X)}=\emptyset\right\}$ and $A=\left\{y \in X, \omega_{f}(y) \subset \overline{\mathcal{M}_{A F}(X)}\right\}$. Clearly, $A \subsetneq X$, for instance, $N \cap A=\emptyset$.

Claim.1: $A$ is a closed strongly invariant subset of $X$.

The claim follow immediately from Theorem 4.3 and the definition of $A$. Indeed let $\left(y_{n}\right)_{n \geq 0}$ a sequence of $A$ converging to some $y \in X$. If $\omega_{f}(y)$ is finite, then it is a periodic orbit, thus $y \in A$. If not $\omega_{f}(y)$ is infinite, then by Theorem 4.3, $\left(\omega_{f}\left(y_{n}\right)\right)_{n \geq 0}$ converges to $\omega_{f}(y)$ and since for any $n \geq 0, \omega_{f}\left(y_{n}\right) \subset \overline{\mathcal{M}_{A F}(X)}$, then $\omega_{f}(y) \subset \overline{\mathcal{M}_{A F}(X)}$. Thus $y \in A$. 
By Lemma 2.8, we may then collapse $A$ to a fixed point. Denote by $\phi$ the quotient map and consider $g$ the induced homeomorphism. Clearly $\phi(X)=\left(\bigcup_{M \in \mathcal{N}}^{\emptyset} \phi(A(M))\right) \bigcup^{\emptyset} \phi(A)$.

Observe that $g$ has an unique periodic point $a(\phi(A))$, in fact for any $x \notin A, \omega_{f}(x)$ is infinite minimal set such that $\omega_{f}(x) \cap \overline{\mathcal{M}_{A F}(X)}=\emptyset$, thus $\phi\left(\omega_{f}(x)\right)=\omega_{g}(\phi(x))$ is an infinite minimal set for $g$. Therefore for any $\phi(x) \neq a, \omega_{g}(\phi(x)) \neq\{a\}$. Notice that for any $x \in X \backslash A$, the map $\phi: A\left(\omega_{f}(x)\right) \rightarrow \phi\left(A\left(\omega_{f}(x)\right)\right)=A\left(\omega_{g}(\phi(x))\right)$ is an homeomorphism, thus the set $A\left(\omega_{g}(\phi(x))\right)$ has infinitely many connected component for any $x \in X \backslash A$. So we may assume that $P(f)=\{a\}$, where $a$ is a fixed point for $f$ and for any $x \neq a, \omega_{f}(x)$ is an infinite minimal subset for which the set $A\left(\omega_{f}(x)\right)$ has infinitely many connected component.

Claim.2: The map $\omega_{f}$ is uniformly continuous on $X$.

The continuity of the map $\omega_{f}$ on $X \backslash\{a\}$ follow from Theorem 4.3. $X$ is a compact metric space, then it will be enough to prove that the map $\omega_{f}$ is also continuous at $a$, this is a consequence for Theorem 4.7 and the fact that $a$ is a distal point and the unique periodic point. In fact, let $\left(a_{n}\right)_{n \geq 0}$ a sequence of $X$ converging to $a$, we may assume that for any $n \geq 0, a_{n} \neq a$. Thus $\omega_{f}\left(a_{n}\right)$ is infinite, by Lemma 4.6 , for any $n \geq 0, \omega_{f}\left(a_{n}\right)=\alpha_{f}\left(a_{n}\right)$. By Lemma 4.7 , one of the sequences $\left(\omega_{f}\left(a_{n}\right)\right)_{n \geq 0}$ or $\left(\alpha_{f}\left(a_{n}\right)\right)_{n \geq 0}$ converges to $\omega_{f}(a)=\{a\}$. Thus the map $\omega_{f}$ is also continuous on $a$, hence uniformly continuous on $X$.

Since any minimal set $M \neq\{a\}$ is infinite it follow by Theorem 5.1 that $A(M)$ is closed, moreover observe that $A(\{a\})=\{a\}$. Hence for any minimal set for $(X, f), A(M)$ is a closed set. Clearly $X=\bigcup_{i \in I}^{\emptyset} A\left(M_{i}\right)$, where $\left\{M_{i}, \quad i \in I\right\}$ is the set of minimal subset of $(X, f)$. Since the family $\left(A\left(M_{i}\right)\right)_{i \in I}$ is pairwise disjoint, we may define the equivalence relation $\mathcal{R}$ that collapse each connected component of each $A\left(M_{i}\right)$ to a point. Denote by $\pi$ the naturel quotient map.

Claim 3: The quotient space $\widetilde{X}$ is a hereditarily locally connected continuum.

Let $G$ be the graph of $\mathcal{R}$. We prove first that $G$ is closed, indeed let $\left(x_{n}, y_{n}\right) \in G$ such that $\left(x_{n}, y_{n}\right)$ converges to $(x, y)$.

Let $C_{n}$ be the connected component of $A\left(M_{n}\right)$ that contains $\left\{x_{n}, y_{n}\right\}$. By claim 2, the map $\omega_{f}$ is continuous, it follow immediately that any limit point of $\left(C_{n}\right)_{n \geq 0}$ namely $C$ is a connected subset of $X$ containing the set $\{x, y\}$, moreover $C \subset A\left(\omega_{f}(x)\right)=A\left(\omega_{f}(y)\right)$, thus $(x, y) \in G$. Therefore the result follow. By [13], Proposition 3.7 and Theorem 3.10, since $G$ is closed, $\widetilde{X}$ is a continuum and clearly $\pi$ is monotone, thus $\widetilde{X}$ is a hereditarily locally 
connected.

$$
\text { Claim.4: } \pi(X)=\pi\left(\bigcup_{i \in I}^{\emptyset} M_{i}\right)=\pi(\Omega(f)) \text {. }
$$

By Lemma 5.12, for any minimal set $M$, any connected component of $A(M)$ meets $M$, therefore $\pi(A(M))=\pi(M)$. Recall that $X=\bigcup_{i \in I}^{\emptyset} A\left(M_{i}\right)$, thus $\pi(X)=\pi\left(\bigcup_{i \in I}^{\emptyset} M_{i}\right)$. By Theorem 3.3, we have $\Omega(f)=A P(f)=\bigcup_{i \in I}^{\emptyset} M_{i}$ and the result follow.

Note that for any $i \in I$ and for any $C_{i}$ a connected component of $A\left(M_{i}\right), f\left(C_{i}\right)$ and $f^{-1}\left(C_{i}\right)$ are also connected components of $A\left(M_{i}\right)$, therefore $\pi$ induce naturally an homeomorphism $g$ on $\widetilde{X}$ satisfying $g(\pi(x))=$ $\pi(f(x))$.

Observe that by claim $3, g$ is an homeomorphism on the hereditarily locally connected continuum $\widetilde{X}$ and satisfy the follow :

(i) $A P(g)=\Omega(g)=\tilde{X}$, this follow from claim $4, \widetilde{X}=\bigcup_{i \in I}^{\emptyset} \pi\left(M_{i}\right)$, where for each $i \in I, \pi\left(M_{i}\right)$ is a minimal set for $(\widetilde{X}, g)$.

(ii) The map $\omega_{g}: \widetilde{X} \rightarrow 2^{\widetilde{X}}$ is uniformly continuous, this follow from (i) and Corollary 4.2 .

(iii) Observe that $\pi(x) \in P(g)$ if and only if $A\left(\omega_{f}(x)\right)$ has a finite number of connected component, therefore $P(g)=\{\widetilde{a}\}$, where $\tilde{a}=\pi(a) \in \widetilde{X}$.

Claim.5: $(\tilde{X}, g)$ is equicontinoues.

By Lemma 2.1, it will be enough to prove that $R P(g)=\Delta_{\tilde{X}}$. Let $x \in X$, by Lemma $2.2, R P_{\tilde{x}}(g)$ is a subcontinuum of $\tilde{X}$. We are going to prove first that $R P_{\tilde{x}}(g) \subset \omega_{g}(\tilde{x})$, so let $\tilde{y} \in R P_{\tilde{x}}(g)$.

We claim that $\omega_{g}(\tilde{y})=\omega_{g}(\tilde{x})$. Indeed since $\tilde{y} \in R P_{\widetilde{x}}(g)$, we can find two sequences of $\tilde{X},\left(\tilde{x}_{n}\right)_{n \geq 0},\left(\tilde{y}_{n}\right)_{n \geq 0}$ converging respectively to $\tilde{x}, \tilde{y}$ and a sequence of integer $\left(m_{n}\right)_{n \geq 0}$ such that $\lim _{n \rightarrow+\infty} d\left(g^{m_{n}}\left(\tilde{x}_{n}\right), g^{m_{n}}\left(\tilde{y}_{n}\right)\right)=0$, thus by (ii), since the map $\omega_{g}$ is uniformly continuous:

$$
\lim _{n \rightarrow+\infty} d_{H}\left(\omega_{g}\left(g^{m_{n}}\left(\tilde{x}_{n}\right)\right), \omega_{g}\left(g^{m_{n}}\left(\tilde{y}_{n}\right)\right)\right)=0 .
$$

For any $n \geq 0, d_{H}\left(\omega_{g}\left(g^{m_{n}}\left(\widetilde{x}_{n}\right)\right), \omega_{g}\left(g^{m_{n}}\left(\tilde{y}_{n}\right)\right)\right)=d_{H}\left(\omega_{g}\left(\tilde{x}_{n}\right), \omega_{g}\left(\tilde{y}_{n}\right)\right)$. Thus $\omega_{g}(\tilde{x})=\omega_{g}(\tilde{y})$ and by (i) since $A P(g)=\Omega(g)=\tilde{X}$, it follow that $\tilde{y} \in \omega_{g}(\tilde{x})$. Therefore $R P_{\tilde{x}}(g) \subset \omega_{g}(\tilde{x})$, thus $\pi^{-1}\left(R P_{\tilde{x}}(g)\right) \subset \pi^{-1}\left(\omega_{g}(\tilde{x})\right) \subset A\left(\omega_{f}(x)\right)$. Recall that the map $\pi$ is monotone and $R P_{\tilde{x}}(g)$ is connected, therefore $\pi^{-1}\left(R P_{\tilde{x}}(g)\right)$ is a connected subset of $X$ contained in $A\left(\omega_{f}(x)\right)$. We conclude that $\pi\left(\pi^{-1}\left(R P_{\tilde{x}}(g)\right)\right)=R P_{\tilde{x}}(g)$ is reduced to a point, therefore $R P_{\widetilde{x}}(g)=$ $\{\tilde{x}\}$ and $R P(g)=\Delta_{\tilde{X}}$. It turn out that $(\tilde{X}, g)$ is equicontinoues. This ends 
the proof of claim 5 .

End of the proof of Lemma 5.6.

As $(\tilde{X}, g)$ is equicontinoues, Then any map in the closure of $\left\{g^{n}, n \in \mathbb{Z}\right\}$ with respect to the uniform metric on $\widetilde{X}^{\widetilde{X}}$ is an homeomorphism. Therefore any two point in the same minimal can be mapped to each other by a self homeomorphism of $\widetilde{X}$. Recall that $\widetilde{X}$ is a hereditarily locally connected continuum, thus by (ii) of Lemma 5.4 the set of local separating point $L(\widetilde{X})$ is dense in $\tilde{X}$. Note that $g$ has a unique fixed point $\tilde{a}$, therefore there exist $\tilde{y} \in L(\tilde{X})$ such that $\tilde{y} \neq \tilde{a}$, recall that $\tilde{y} \in \omega_{g}(\tilde{y})$ which is infinite. Thus $\omega_{g}(\tilde{y}) \subset L(\tilde{X})$. Let $C$ be the connected component of $\widetilde{X} \backslash \omega_{f}(\tilde{y})$ that contains $\tilde{a}$. Clearly $\bar{C} \backslash C=\omega_{g}(\tilde{y})$. As $C$ is a connected set of $\widetilde{X}$, then by (i) of Lemma 5.4, $\bar{C} \backslash C$ contains at most a countable point of local separating, thus the minimal set $\omega_{f}(\tilde{y})$ is countable which is a contradiction.

Theorem 5.7. Let $f: X \rightarrow X$ be a hereditarily locally connected continuum homeomorphism such that $P(f) \neq \emptyset$ and $M$ an infinite minimal set for $(X, f)$, then the following hold :

(i) There exist a sequence $\left(C_{n}\right)_{n \geq 0}$ of subcontinua of $X$ such that:

- $C_{n} \in P\left(2^{f}\right)$, for any $n \geq 0$;

- $C_{n} \cap A(M)=\emptyset$, for any $n \geq 0$;

- $\left(O P_{f}\left(C_{n}\right)\right)_{n \geq 0}$ converges to $M$, with respect to the Hausdroof metric.

(ii) For $t \in M$, there exist a positive sequence of integer $\left(m_{n}\right)_{n \geq 0}$ such that $f^{m_{n}}\left(C_{n}\right)$ converges to $\{t\}$.

For the proof we need the following lemma :

Lemma 5.8. Let $f: X \rightarrow X$ be a hereditarily locally connected continuum homeomorphism such that $P(f) \neq \emptyset$ and $M$ an infinite minimal set for $(X, f)$, then $\operatorname{int}_{\Omega(f)}(M)=\emptyset$.

Proof. Let $M$ be an infinite minimal set, then by Theorem 5.1, $A(M)$ is a closed set, moreover $P(f) \neq \emptyset$, thus $A(M)$ is proper closed subset of $X$ which is connected, then $A(M)$ is not open, so we can find a sequence $\left(x_{n}\right)_{n \geq 0}$ of $X \backslash A(M)$ such that $\left(x_{n}\right)_{n \geq 0}$ converges to some $x \in A(M)$. Since $x \notin P(f)$, then the map $\omega_{f}$ is continuous at $x$, thus the sequence $\omega_{f}\left(x_{n}\right)$ converges to $\omega_{f}(x)=M$. Observe that for any $n \geq 0, \omega_{f}\left(x_{n}\right) \neq M$, therefore by minimality of $M$ and any $\omega$-limit set (see Theorem 3.1), we get $M \cap \omega_{f}\left(x_{n}\right)=\emptyset$, for any $n \geq 0$. Therefore if $\operatorname{int}_{\Omega(f)}(M) \neq \emptyset$, then for some open $O$ of $\Omega(f)$, and for $n$ large enough, we get $\omega_{f}\left(x_{n}\right) \cap O \neq \emptyset$ and $O \subset M$, which will lead to a contradiction.

Proof of Theorem 5.7 (i) Let $x \in M$, by Lemma 5.6, there exists $\left(x_{n}\right)_{n \geq 0}$ a sequence of $\mathcal{M}_{A F}(X)$ converging to $x$, moreover by Lemma 5.8, since $P(f) \neq \emptyset$, we may assume that for any $n \geq 0, x_{n} \notin M$, thus $\omega_{f}\left(x_{n}\right) \cap M=\emptyset$ 
and by passing to a subsequence if needed, the family $\left(A\left(\omega_{f}\left(x_{n}\right)\right)\right)_{n \geq 0}$ is pairwise disjoints and for any $n \geq 0, A(M) \cap A\left(\omega_{f}\left(x_{n}\right)\right)=\emptyset$.

Case. 1 For infinitely many $n \geq 0, x_{n} \in P(f)$.

In this case let $C_{n}:=x_{n}$. Clearly $\left(C_{n}\right)_{n \geq 0}$ is a sequence of subcontinua of $X$, moreover by Theorem 4.3, the map $\omega_{f}$ is continuous at $x$, thus the sequence $\left(\omega_{f}\left(x_{n}\right)\right)_{n \geq 0}$ converges to $\omega_{f}(x)=M$ and since $x_{n} \in P(f)$, then $\omega_{f}\left(x_{n}\right)=O_{f}\left(x_{n}\right)$ and $x_{n} \notin A(M)$. This end the proof of this case.

Case.2 For $n$ large enough, $x_{n} \notin P(f)$, thus $\omega_{f}\left(x_{n}\right)$ is infinite.

Denote by $M_{n}=\omega_{f}\left(x_{n}\right)$ and $M=\omega_{f}(x)$, by same argument of the first case, the sequence $\left(M_{n}\right)_{n>0}$ converges to $M$.

Since for any $n \geq 0, M_{n}$ is infinite, then $A\left(M_{n}\right)$ is a closed subset of $X$.

Claim 1: For any sequence $\left(y_{n}\right)_{n \geq 0}$ for which $y_{n} \in A\left(M_{n}\right)$, any limit point of $\left(y_{n}\right)_{n \geq 0}$ is a point of $A(M)$.

Indeed let $y_{n} \in A\left(M_{n}\right)$ such that $\left(y_{n}\right)_{n \geq 0}$ converge to some $y \in X$. Therefore by Lemma 4.7, at least one of the sequence $\left(\alpha_{f}\left(y_{n}\right)\right)_{n \geq 0}$ or $\left(\omega_{f}\left(y_{n}\right)\right)_{n \geq 0}$ converges to $\omega_{f}(y)$. Recall that for any $n \geq 0, \omega_{f}\left(y_{n}\right)=M_{n}$ is infinite therefore by Lemma 4.6 , for any $n \geq 0, \alpha_{f}\left(y_{n}\right)=\omega_{f}\left(y_{n}\right)=M_{n}$. We conclude that $\omega_{f}(y)=\lim _{n \rightarrow+\infty} M_{n}=M$, thus $y \in A(M)$. This end the proof of the claim.

So let $y_{n} \in A\left(M_{n}\right)$, so that $\left(y_{n}\right)_{n \geq 0}$ converges to $y \in A(M)$ and let $C_{n}$ be the connected component of $A\left(M_{n}\right)$ that contains $y_{n}$. Since $M_{n} \in \mathcal{M}_{A F}$, then for any $n \geq 0$, by Lemma 5.5, $C_{n} \cap M_{n} \neq \emptyset$ and $C_{n} \in P\left(2^{f}\right)$, moreover $O P_{f}\left(C_{n}\right) \subset A\left(M_{n}\right)$.

Claim 2.: The family $\mathcal{C}=\left\{f^{m}\left(C_{n}\right), n \geq 0, m \in \mathbb{Z}\right\}$ is a null family.

If not we may find some $\eta>0$ and $D_{m} \in \mathcal{C}$ such that for any $m \geq$ $0, \operatorname{diam}\left(C_{m}\right)>\eta$. Since for any $n \geq 0, C_{n} \in P\left(2^{f}\right)$, we may assume that $D_{m}=f^{s_{m}}\left(C_{n_{m}}\right)$, where $\left(s_{m}\right)_{m>0}$ and $\left(n_{m}\right)_{m>0}$ are sequences of integers such that $\left(n_{m}\right)_{m>0}$ is increasing and $\left(D_{m}\right)_{m>0}$ converges to some $D \in C(X)$, with respect to the Hausdroof metric. Obviously $\operatorname{diam}(D) \geq \eta$ and by Claim 1, $D \subset A(M)$. Recall that the minimal sets $\left(M_{n}\right)_{n \geq 0}$ are disjoints from $M$, therefore their respective sets $\left(A\left(M_{n}\right)\right)_{n \geq 0}$ are disjoints with $A(M)$, we conclude that $D \cap D_{m}=\emptyset$, for any $m \geq 0$. It turn out that $D$ is a convergence continuum of $X$. A contradiction with Lemma 2.5, this end the proof of claim 2 .

Now since $\mathcal{C}$ is a null family, each element of which meets some $M_{n}$, we conclude that for any converging sequence of elements of $\mathcal{C}$, its limit with respect to the Hausdroof limit must be reduced to $\{t\}$, where $t \in M$.

End of the proof of Theorem 5.7. Since $2^{X}$ is a compact set, we can assume that the sequence $\left(O P_{f}\left(C_{n}\right)\right)_{n \geq 0}$ converges. By claim 1, the sequence $\left(O P_{f}\left(C_{n}\right)\right)_{n \geq 0}$ converges to $N \subset A(M)$ and by claim 2 , the sequence $\left(O P_{f}\left(C_{n}\right)\right)_{n \geq 0}$ converges to $N \subset M$. Observe that $f(N)=N$, therefore by minimality of $M$, we get $M=N$. Recall that the family $\left(A\left(\omega_{f}\left(x_{n}\right)\right)\right)_{n \geq 0}$ is pairwise disjoints and for any $n \geq 0, A(M) \cap A\left(\omega_{f}\left(x_{n}\right)\right)=\emptyset$. Moreover $C_{n} \subset A\left(M_{n}\right)$ is a connected component of $A\left(M_{n}\right)$ where $M_{n} \in \mathcal{M}_{A F}$, this 
ends the proof of (i).

(ii) It will be enough to prove that for any $t \in M$ and for any neighbourhood $O_{t}$ of $t$, we may find some $m_{n}>0$ such that $f^{m_{n}}\left(C_{n}\right) \subset O_{t}$. So let $O_{t}$ be some neighbourhood of $t$. Since the sequence $\left(O P_{f}\left(C_{n}\right)\right)_{n \geq 0}$ converges to $M$ and $t \in M$, we may find for each $n \geq 0$ some $t_{n} \in O P_{f}\left(C_{n}\right)$, so that $\left(t_{n}\right)_{n \geq 0}$ converges to $t$. We may assume that for any $n \geq 0, t_{n} \in O_{t}$. Let $m_{n} \geq 0$ so that $t_{n} \in f^{m_{n}}\left(C_{n}\right)$, recall that $\mathcal{C}$ is a null family (claim 2 of the proof of (i)), therefore $\lim _{n \rightarrow+\infty} \operatorname{diam}\left(f^{m_{n}}\left(C_{n}\right)\right)=0$. Denote by $\gamma=\frac{d\left(t, \partial\left(O_{t}\right)\right)}{2}$, clearly $\gamma>0$. Assume that for infinitely many $n \geq 0, f^{m_{n}}\left(C_{n}\right) \nsubseteq O_{t}$, therefore $f^{m_{n}}\left(C_{n}\right) \cap \partial\left(O_{t}\right) \neq \emptyset\left(f^{m_{n}}\left(C_{n}\right)\right.$ is a connected subset of $X$ meeting $O_{t}$ at $t_{n}$ and $\left.f^{m_{n}}\left(C_{n}\right) \nsubseteq O_{t}\right)$, therefore for $n$ large enough we get $\operatorname{diam}\left(f^{m_{n}}\left(C_{n}\right)\right)>$ $\gamma$, a contradiction, we conclude that for some $n \geq 0, f^{m_{n}}\left(C_{n}\right) \subset O_{t}$, this ends the proof of (ii).

Theorem 5.7, prove that if we allow periodic point for hereditarily locally connected continuum homeomorphism, any infinite minimal set is the Hausdroof limit, of a null sequence of periodic cycle, this is a weaker version of Theorem 5.3 but it will enable us to prove that infinite minimal satisfy the following :

(i) $M$ is not totally minimal for any iterate of $f$, Lemma 5.9.

(ii) $M$ is distal, Lemma 5.10 .

(iii) $M$ is totaly disconnected, Lemma 5.11 .

After we establish (i),(ii) and (iii), we prove Theorem 5.3

Lemma 5.9. Let $f: X \rightarrow X$ be a hereditarily locally connected continuum homeomorphism such that $P(f) \neq \emptyset$ and $M$ an infinite minimal set for $(X, f)$, then $M$ is not totaly minimal.

Proof. Let $M$ be an infinite minimal set for $(X, f)$. By Theorem 5.1, $A(M)$ is a closed set. Assume that $M$ is totaly minimal, therefore for any $x \in A(M)$ and for any $n \in \mathbb{Z}, \omega_{f^{n}}(x)=M$. Since $P(f) \neq \emptyset$, then $A(M)$ is a proper closed subset of $X$, so let $a \neq b \in M$, denote by $\eta=d(a, b)>0$. By Lemma 2.10 let $U$ an open neighbourhood of $A(M)$ such that $M e s h(U \backslash A(M))<\frac{\eta}{2}$. By Theorem 5.7, we may find $\left(C_{n}\right)_{n \geq 0}$ a sequence of continua, for which their orbit form a periodic cycle of length $p_{n}$ such that $\lim _{n \rightarrow+\infty} C_{n}=\{a\}$ and $C_{n} \cap A(M)=\emptyset$. Therefore we can find a sequence of continua $\left(D_{n}\right)_{n \geq 0}$ such that for any $n \geq 0 C_{n} \cup\{a\} \subset D_{n}$ and $\lim _{n \rightarrow+\infty} D_{n}=\{a\}$. For each $n \geq 0$, consider $T_{n}$ the connected component of $C_{n}$ in $D_{n} \backslash A(M)$, clearly $\overline{T_{n}} \cap A(M) \neq \emptyset$, so let $a_{n} \in \overline{T_{n}} \cap A(M)$, observe that $I_{n}=T_{n} \cup\left\{a_{n}\right\}$ is a connected subset of $X$ and $I_{n} \backslash A(M)=T_{n}$ is connected.

Recall that for any $m, n \geq 0, \omega_{f m}\left(a_{n}\right)=M$ and $b \in M$, therefore for each $n \geq 0$, we can find some $s_{n}>0$ so that $\left(f^{p_{n} s_{n}}\left(a_{n}\right)\right)_{n \geq 0}$ converges to $b$. For any $n \geq 0, f^{p_{n} s_{n}}\left(C_{n}\right)=C_{n}$, therefore $f^{p_{n} s_{n}}\left(I_{n}\right)$ is a connected subset of $X$ that contains $C_{n}$ (resp. $f^{p_{n} s_{n}}\left(a_{n}\right)$ ) and it converges to $\{a\}$ (resp. 
b), therefore for $n$ large enough, we have $\operatorname{diam}\left(f^{p_{n} s_{n}}\left(I_{n}\right)\right)>\frac{\eta}{2}$, moreover $f^{p_{n} s_{n}}\left(I_{n}\right) \backslash A(M)=f^{p_{n} s_{n}}\left(T_{n}\right)=f^{p_{n} s_{n}}\left(I_{n}\right) \backslash\left\{f^{p_{n} s_{n}}\left(a_{n}\right)\right\}$ is connected, thus for any $n \geq 0, f^{p_{n} s_{n}}\left(I_{n}\right) \not \subset U$, hence it meets $\partial(U)$, so denote by $t_{n}$ some point of $f^{p_{n} s_{n}}\left(I_{n}\right) \cap \partial(U)$, hence $f^{-p_{n} s_{n}}\left(t_{n}\right) \in I_{n}$, therefore the sequence $\left(f^{-p_{n} s_{n}}\left(t_{n}\right)\right)_{n \geq 0}$ converges to $a$. By Theorem, 4.3 , since $a \notin P(f)$, the sequence $\left(\omega_{f}\left(f^{-p_{n} s_{n}}\left(t_{n}\right)\right)\right)_{n>0}\left(\operatorname{resp}\left(\alpha_{f}\left(f^{-p_{n} s_{n}}\left(t_{n}\right)\right)\right)_{n>0}\right)$ converges to $\omega_{f}(x)=M\left(\operatorname{resp} . \alpha_{f}(x)=M\right)$, therefore $\left(\omega_{f}\left(t_{n}\right)\right)_{n \geq 0}\left(\operatorname{resp} . \quad\left(\alpha_{f}\left(t_{n}\right)\right)_{n \geq 0}\right)$ also converges to $M$.

Let $t \in \partial(U)$, be the limit of the sequence $\left(t_{n}\right)_{n \geq 0}$. By Lemma 4.7, at least one of the sequence $\left(\omega_{f}\left(t_{n}\right)\right)_{n \geq 0}$ or $\left(\alpha_{f}\left(t_{n}\right)\right)_{n \geq 0}$ converges to $\omega_{f}(t)$ and since both of them converges to $M$ we conclude that $\omega_{f}(t)=M$, therefore $t \in A(M) \cap \partial(U)$, this will lead to a contradiction since $A(M) \subset U$ and $U$ is an open set.

Lemma 5.10. Let $f: X \rightarrow X$ be a hereditarily locally connected continuum homeomorphism such that $P(f) \neq \emptyset$ and $M$ an infinite minimal set for $(X, f)$, then $M$ is distal.

Proof. Let $M$ be some infinite minimal set for $(X, f)$ and $a \neq b$ such that $(a, b) \in P(M, f)$, observe that for any $n \geq 0, b \in \omega_{f^{n}}(a)$, otherwise $(a, b)$ will be a distal pair for some $f^{m}$, thus distal for $f$. Denote by $\eta=d(a, b)>0$. by Theorem 5.1, $A(M)$ is a closed set, moreover it is a proper subset of $X$ since $P(f) \neq \emptyset$. By Lemma 2.10 let $U$ an open neighbourhood of $A(M)$ such that $\operatorname{Mesh}(U \backslash A(M))<\frac{\eta}{2}$.

By Theorem 5.7, we may find $\left(C_{n}\right)_{n \geq 0}$ a sequence of continua, for which their orbit form a periodic cycle of length $p_{n}$ such that $\lim _{n \rightarrow+\infty} C_{n}=\{a\}$ and $C_{n} \cap A(M)=\emptyset$. So denote by $D_{n}$ the connected component of $X \backslash A(M)$ that contains $C_{n}$, observe that $f^{p_{n}}\left(D_{n}\right)=D_{n}$ and $\overline{D_{n}} \cap A(M) \neq \emptyset$, therefore by minimality of $M$, for each $n \geq 0$, we get $M=\bigcup_{0 \leq i \leq p_{n}} f^{i}\left(\overline{D_{n}}\right) \cap M$. For each $n \geq 0$, let $i_{n} \leq p_{n}$ so that $a \in f^{i_{n}}\left(\overline{D_{n}}\right) \cap M$.

Claim: There exist $\left(V_{n}\right)_{n \geq 0}$ is a sequence of connected subset of $X$ such that:

(i) $V_{n} \cap A(M)=\{a\}$ and $V_{n} \backslash A(M)$ is connected.

(ii) $V_{n}$ contains $\{a\}$ and $f^{j_{n}}\left(C_{n}\right)$, where $\left(f^{j_{n}}\left(C_{n}\right)\right)_{n \geq 0}$ converges to $\{a\}$ with respect to the Hausdroof metric.

(iii) $\left(\overline{V_{n}}\right)_{n \geq 0}$ converges to $\{a\}$ with respect to the Hausdroof metric.

Observe that the family $\left\{f^{i_{n}}\left(D_{n}\right), n \geq 0\right\}$ is a sub-family of those of the connected component of $X \backslash A(M)$, therefore it is either finite or a null.

Case 1: The family $\left\{f^{i_{n}}\left(D_{n}\right), n \geq 0\right\}$ is finite.

If the family $\left\{f^{i_{n}}\left(D_{n}\right), n \geq 0\right\}$ is finite, then we may assume that for any $n \geq$ $0, f^{i_{n}}\left(D_{n}\right)=f^{i_{0}}\left(D_{0}\right)$, hence $D_{n} \subset f^{i_{0}-i_{n}}\left(D_{0}\right)$, recall that $f^{p_{0}}\left(D_{0}\right)=D_{0}$, hence by passing to a subsequence if needed, for some $0 \leq k \leq p_{0}, f^{i_{0}-i_{n}}\left(D_{0}\right)=$ $f^{k}\left(D_{0}\right)$, for any $n \geq 0$. Thus $D_{n} \subset f^{k}\left(D_{0}\right)$. Therefore, for any $n \geq 0, C_{n} \subset$ $D_{n} \subset f^{k}\left(D_{0}\right)$. Since $\lim _{n \rightarrow+\infty} C_{n}=\{a\}$, then $f^{k}\left(D_{0}\right) \cup\{a\}$ is connected hence locally connected since $X$ is a hereditarily locally connected continuum and 
contains all the $\left(C_{n}\right)_{n \geq 0}$. For each $n \geq 0$, let $\widetilde{V_{n}}$ some connected neighbourhood of $a$ in $f^{k}\left(D_{0}\right) \cup\{a\}$, so that $\left(\overline{\widetilde{V_{n}}}\right)_{n \geq 0}$ converges to $\{a\}$ with respect to the Hausdroof metric, since $f^{k}\left(D_{0}\right) \cup\{a\}$ contains all the $C_{n}$, me may assume that for any $n \geq 0, C_{n} \cup\{a\} \subset \widetilde{V_{n}}$. Note that $\widetilde{V_{n}}$ is also locally connected since it is a connected subset of $X$ which is hereditarily locally connected. For any $n \geq 0$, let $T_{n}$ be the connected component of $\widetilde{V_{n}} \backslash\{a\}$ that contains $C_{n}$, by (iii) of Lemma 2.3 , we have $a \in B d_{\widetilde{V}_{n}}\left(T_{n}\right)$, therefore $V_{n}=T_{n} \cup\{a\}$ is a connected subset of $\widetilde{V_{n}}$.

Observe that $V_{n} \cap A(M)=\{a\}$ and $V_{n} \backslash A(M)=T_{n}$ thus connected, this prove (i). Recall that $V_{n} \subset \widetilde{V_{n}}$ and ${\widetilde{V_{n}}}_{n \geq 0}$ converges to $\{a\}$, this prove (ii). For (iii) it will be enough to pick $j_{n}=0$ for any $n \geq 0$.

Case 2: The family $\left\{f^{i_{n}}\left(D_{n}\right), n \geq 0\right\}$ is infinite, thus a null family.

If $\left\{f^{i_{n}}\left(D_{n}\right), n \geq 0\right\}$ is a null family we may pick $V_{n}=f^{i_{n}}\left(D_{n}\right) \cup\{a\}$, recall that $a \in \overline{f^{i_{n}}\left(D_{n}\right)}$ and $f^{i_{n}}\left(D_{n}\right)$ is connected, thus $V_{n}$ is a connected subset of $X$, moreover $V_{n} \backslash A(M)=f^{i_{n}}\left(D_{n}\right)$, which is also connected, thus prove (i). Since $\lim _{n \rightarrow+\infty} \operatorname{diam}\left(V_{n}\right)=0$, then $\left(\overline{V_{n}}\right)_{n \geq 0}$ converges to $\{a\}$ with respect to the Hausdroof metric, and $f^{i_{n}}\left(C_{n}\right) \cup\{a\} \subset V_{n}$, this prove (ii) and (iii) where $j_{n}=i_{n}$. This end the proof of the claim.

End of the proof of Lemma 5.10: Recall that $f^{p_{n}}\left(C_{n}\right)=C_{n}$ and for any $n \geq 0, b \in \omega_{f^{n}}(a)$, hence for any $n \geq 0$, we may find some $s_{n}>0$ so that $\left(f^{p_{n} s_{n}}(a)\right)_{n \geq 0}$ converges to $b$. Thus for any $n \geq 0, f^{p_{n} s_{n}}(a) \in f^{p_{n} s_{n}}\left(V_{n}\right)$ and $f^{j_{n}}\left(C_{n}\right)=f^{j_{n}+p_{n} s_{n}}\left(C_{n}\right) \subset f^{p_{n} s_{n}}\left(V_{n}\right)$, where $\left(f^{j_{n}}\left(C_{n}\right)\right)_{n \geq 0}$ converges to $\{a\}$ with respect to the Hausdroof metric. Therefore for $n$ large enough we have $\operatorname{diam}\left(f^{p_{n} s_{n}}\left(V_{n}\right)\right)>\frac{\eta}{2}$. Recall that $V_{n} \cap A(M)=\{a\}$ and $V_{n} \backslash A(M)$ is connected, then $f^{p_{n} s_{n}}\left(V_{n}\right) \backslash A(M)=f^{p_{n} s_{n}}\left(V_{n}\right) \backslash\left\{f^{p_{n} s_{n}}(a)\right\}$ is also connected. Therefore for any $n \geq 0, f^{p_{n} s_{n}}\left(V_{n}\right) \nsubseteq U$, hence it meets $\partial(U)$, so denote by $t_{n}$ some point of $f^{p_{n} s_{n}}\left(V_{n}\right) \cap \partial(U)$, then $f^{-p_{n} s_{n}}\left(t_{n}\right) \in V_{n}$, therefore the sequence $\left(f^{-p_{n} s_{n}}\left(t_{n}\right)\right)_{n \geq 0}$ converges to $a$. Since $a \notin P(f)$, by Theorem 4.3, the sequence $\left(\omega_{f}\left(f^{-p_{n} s_{n}}\left(t_{n}\right)\right)\right)_{n \geq 0}\left(\operatorname{resp}\left(\alpha_{f}\left(f^{-p_{n} s_{n}}\left(t_{n}\right)\right)\right)_{n \geq 0}\right)$ converges to $\omega_{f}(a)=M\left(\operatorname{resp} . \alpha_{f}(a)=M\right)$, hence $\left(\omega_{f}\left(t_{n}\right)\right)_{n \geq 0}\left(\right.$ resp. $\left.\left(\alpha_{f}\left(t_{n}\right)\right)_{n \geq 0}\right)$ also converges to $M$.

Let $t \in \partial(U)$, be the limit of the sequence $\left(t_{n}\right)_{n \geq 0}$. By Lemma 4.7, at least one of the sequence $\left(\omega_{f}\left(t_{n}\right)\right)_{n \geq 0}$ or $\left(\alpha_{f}\left(t_{n}\right)\right)_{n \geq 0}$ converges to $\omega_{f}(t)$ and since both of them converges to $M$ we conclude that $\omega_{f}(t)=M$, therefore $t \in A(M) \cap \partial(U)$, this will lead to a contradiction since $A(M) \subset U$ and $U$ is an open subset of $X$. We conclude that $M$ has to be distal.

With Lemma 5.9, and Lemma 5.10, we can conclude the third lemma 5.11

Lemma 5.11. Let $f: X \rightarrow X$ be a hereditarily locally connected continuum homeomorphism such that $P(f) \neq \emptyset$ and $M$ an infinite minimal set for $(X, f)$, then $M$ is totaly disconnected, thus a Cantor set.

Proof. Let $M$ be some infinite minimal set for $(X, f)$ and $J$ be some non degenerated connected component of $M$. Observe that the family $\left\{f^{n}(J), n \in\right.$ $\mathbb{Z}\}$ is a null family. In fact $\left(f^{n}(J)\right)_{n \in \mathbb{Z}}$, is pairwise disjoint, indeed if not then 
$f^{k}(J)=J$, for some $k \in \mathbb{Z}$, therefore by simple argument of connectedness $J$ is totaly minimal for $f^{k}$, thus $\left(X, f^{k}\right)$ has a totaly minimal infinite minimal set, while also having periodic point, this contradict Lemma 5.9. We conclude that $\left\{f^{n}(J), n \in \mathbb{Z}\right\}$ is an infinite sub-family of those of the connected component of $M$, therefore by Lemma 2.7 , the family $\left\{f^{n}(J), n \in \mathbb{Z}\right\}$ is a null family. Pick any $a \neq b$ two point in $J$, since $\left\{f^{n}(J), n \in \mathbb{Z}\right\}$ is a null family, then $(a, b)$ is a proper proximal pair for $(M, f)$, this contradict Lemma 5.10. We conclude then that $M$ is a Cantor set.

We will end this subsection, by proving Theorem 5.3, before we do we establish the following lemma:

Lemma 5.12. Let $f: X \rightarrow X$ be a hereditarily locally connected continuum homeomorphism with $P(f) \neq \emptyset$, then for any infinite minimal set $M$, the set $A(M)$ has infinitely many connected component.

Proof. Let $M$ be some infinite minimal set for $(X, f)$ and let $J$ be some connected component of $A(M)$, by Lemma 5.5, $J \cap M \neq \emptyset$, we are going to prove that $J \cap M$ is reduced to a singleton, so assume this is not the case and let $\{a, b\} \subset J \cap M$. Since $J$ is a subcontinuum of $X$ which is hereditarily locally connected, then $J$ is arcwise connected, so let $I$ be some arc of $J$ joining $a, b$. By Lemma $5.11, M$ is a Cantor set, therefore $I \nsubseteq M$ and we can find a sub-arc $K$ of $I$ with end point $c, d$ in $M$ such that $K \backslash\{c, d\} \subset$ $X \backslash \Omega(f)$, therefore $K \subset C$, where $C$ is some connected component of $X \backslash \Omega(f)$, moreover $C \cap A(M) \neq \emptyset$. Since $M$ is infinite, we conclude from (ii) of Lemma 4.5 , that $\bar{C} \times \bar{C} \subset A(X, f)$ hence the pair $(c, d)$ is a proper proximal pair for $(M, f)$, this will contradict for instance Lemma 5.10. It turn out that any connected component of $A(M)$ meets $M$ at one and only one point, therefore $A(M)$ can not have finitely many connected component.

Proof of Theorem 5.3: By Lemma 5.6, $\Omega(f)=\overline{\mathcal{M}_{A F}(X)}$, where $\mathcal{M}_{A F}(X)=$

$\bigcup_{\in \in \mathcal{M}} M$ and $\mathcal{M}_{A F}$ is the set of minimal subset of $(X, f)$ for which $A(M)$ has a finite number of connected component. By Lemma 5.12, it is clear that $\mathcal{M}_{A F}$ is the set of periodic orbit for $(X, f)$, thus the result follow.

\section{Hereditarily locally connected continuum homeomorphism}

This section will be mostly consequences and corollary from the result proven in the previous sections and we try to extend the result proven in $[15],[3]$.

The first result will deal with hereditarily locally connected continuum homeomorphism without periodic points generalising for instance the results in $[15]$.

Theorem 6.1. Let $f: X \rightarrow X$ be a hereditarily locally connected continuum homeomorphism with $P(f)=\emptyset$, then $(X, f)$ has a unique minimal set, which is totaly minimal. 
Proof. Assume that $P(f)=\emptyset$ and $f$ has at least two distinct minimal sets $M$ and $N$, by Lemma 2.8, we may collapse one of them to a fixed point and obtain an homeomorphism $g$ on a hereditarily locally connected continuum for which $P(g)$ is a singleton and having an infinite minimal set, this will contradict Theorem 5.3. Thus $(X, f)$ has an unique minimal set $M$ and by the same argument $M$ is the unique minimal set for any $f^{k}, k \in \mathbb{Z}$, therefore the result follow.

In [2], it was proven that any minimal distal homeomorphism of any zero dimensional compact space is equicontinoues. By ([9], Theorem 4.4) an equicontinoues minimal system on an infinite zero-dimensional compact space is conjugate to an adding machine. Therefore by Lemmas 5.10 and 5.11 , we conclude that any infinite minimal set of a hereditarily locally connected continuum homeomorphism with a non-empty set of periodic points is an adding machine, generalizing for instance the same result for dendrite and regular curve homeomorphism ([3], [1]).

Theorem 6.2. Let $f: X \rightarrow X$ be a hereditarily locally connected continuum homeomorphism with $P(f) \neq \emptyset$. Then each infinite minimal set is an adding machine.

In [3], it was proven that a regular curve homeomorphism with periodic points does not have Li-Yorke pairs. To do so it was proven by using only Theorem 5.3 and Theorem 6.2 in the regular curve case, that any point is approximately periodic. In our setting, those two Theorems are still valid, so the proofs are word by word the ones in [3].

Definition 6.3. Let $X$ be a compact metric space and $f: X \rightarrow X$ be a continuous map. A point $x$ is approximately periodic if for every $\epsilon>0$ there is a periodic point $p$ such that $\lim _{\sup _{n \rightarrow \infty}} d\left(f^{n}(x), f^{n}(p)\right)<\epsilon$.

Theorem 6.4. Every point is approximately periodic for any hereditarily locally connected continuum homeomorphism with a non-empty set of periodic points.

Corollary 6.5. There is no Li-Yorke pair for any hereditarily locally connected continuum homeomorphism with a non-empty set of periodic points.

By a classical result in [5], it is known that if a dynamical system has a positive topological entropy, then it is Li-Yorke chaotic, in particular admit Li-Yorke pairs, thus with corollary 6.5, we conclude the following :

Corollary 6.6. Let $f: X \rightarrow X$ be a hereditarily locally connected continuum homeomorphism with a non-empty set of periodic points, then the topological entropy of $f$ is zero.

Theorem 6.7. Let $f: X \rightarrow X$ be a hereditarily locally connected continuum homeomorphism. Then the following assertions hold:

(i) The dynamical system $\left(\Omega(f), f_{\mid \Omega(f)}\right)$ is equicontinoues if $P(f) \neq \emptyset$.

(ii) The dynamical system $(X, f)$ is equicontinoues if and only if either $(X, f)$ is conjugate to an irrational rotation of the circle or $P(f) \neq \emptyset$ and $\Omega(f)=X$. 
Proof. The proofs are analogue to the one of the same result of the regular curve case in [3], Theorem 5.2.

Acknowledgements. The author is thankful to Mr.Issam Naghmouchi for his helpful remarks and discussion during the preparation of the paper.

\section{REFERENCES}

1. G. Acosta, P. Eslami and L. G. Oversteegen, On open maps between dendrites, Houston J. Math. 33. (3) (2007), 753-770.

2. R. Ellis, Distal transformation groups, Pacific J. Math. 8 (1957), 401-405.

3. A,Daghar. I, Naghmouchi. M, Riahi. Periodic Points of Regular Curve Homeomorphisms. Qual. Theory Dyn. Syst. 20, 32 (2021).

4. A.Daghar. On regular curve homeomorphisms without periodic points. preprint, Journal of Difference Equations and Applications, (doi.org/10.1080/10236198.2021.1912030)

5. Blanchard, F., Glasner, E., Kolyada, S., Maass, A. (2002). On Li-Yorke pairs. Journal fur die reine und angewandte Mathematik, 547, 51-68.

6. E. Glasner and M. Megrelishvili, Group actions on treelike compact spaces. Science. China Mathematics (2019), 62, 2447.

7. W. Huang, S. M. Li, S. SHAO and X.D, YE Null systems and sequence entropy pairs. Ergodic Theory and Dynamical Systems, 23(5),(2003). 1505-1523.

8. K. Kuratowski, Topology Vol. II, Academic Press, New York, (1968).

9. P. Kurka, Topological and Symbolic Dynamics, Société Mathématique de France (2003).

10. D, S, Lipham. Approximations by disjoint continua and a positive entropy conjecture. arXiv preprint arXiv:1910.00563. (2019)

11. C,Mouron. . Mixing sets, positive entropy homeomorphisms and non-Suslinean continua. Ergodic Theory and Dynamical Systems, (2016) 36(7), 2246.

12. T.Mackowiak. Locally weakly confluent mappings on hereditarily locally connected continua. Fund. Math. 88 (1975), no. 3, 225-240

13. S. B. Nadler, Continuum Theory: An Introduction, Monographs and Textbooks in Pure and Applied Mathematics Vol. 158, Marcel Dekker, New York, 1992.

14. I. Naghmouchi, Homeomorphisms of regular curves, J. Difference Equ. Appl., 23 (2017), 1485-1490.

15. I. Naghmouchi, Dynamics of Homeomorphisms of regular curves, Colloquium Math., 162 (2020), 263-277.

16. I.Naghmouchi. Dynamics of monotone graph, dendrite and dendroid maps. International Journal of Bifurcation and Chaos, 21(11), (2011), 3205-3215.

17. M. W. Kincaid. On non-cut sets of locally connected continua. Bulletin of the American Mathematical Society, (1943) 49(6), 399-406.

18. G. T. Seidler, The Topological entropy of homeomorphism on one-dimensional continua. Proc. Amer. Math. Soc. 108 (1990), no. 4, 1025-1030.

19. J.N.Simone. A property of hereditarily locally connected continua related to arcwise accessibility. Journal of the Australian Mathematical Society, 25(1), (1978), 35-40.

20. E.D. Tymchatyn, Some rational continua. Rocky Mountain J. Math. 13 no. 2 (1983) 309-320.

21. Whyburn, G. T. Sets of local separating points of a continuum. Bulletin of the American Mathematical Society, (1933), 39(2), 97-100.

22. Whyburn, G. T. (1948). Analytic topology (Vol. 28). American Mathematical Society. 
HOMEOMORPHISM OF HEREDITARILY LOCALLY CONNECTED CONTINUA 29

Aymen Daghar, University of Carthage, Faculty of SCIEnces of Bizerte, (UR17ES21), "Dynamical Systems and their Applications", 7021, Jarzouna, TUNISIA

Email address: aymendaghar@gmail.com 